\title{
Three-fluorophore FRET enables the analysis of ternary protein association in living plant cells
}

Nina Glöcknera, Sven zur Oven-Krockhaus ${ }^{a, b}$, Leander Rohra ${ }^{a}$, Frank Wackenhut ${ }^{b}$, Moritz Burmeister ${ }^{b}$, Friederike Wanke ${ }^{a}$, Eleonore Holzwart ${ }^{c}$, Alfred J. Meixner ${ }^{b}$, Sebastian Wolf ${ }^{\mathrm{C}}$ and Klaus Harter ${ }^{\mathrm{a}^{*}}$

${ }^{\text {a} C e n t e r ~ f o r ~ P l a n t ~ M o l e c u l a r ~ B i o l o g y, ~ U n i v e r s i t y ~ o f ~ T u ̈ b i n g e n, ~ T u ̈ b i n g e n, ~ G e r m a n y ~}$

bInstitute for Physical \& Theoretical Chemistry, University of Tübingen, Tübingen, Germany

${ }^{\mathrm{c} C}$ Centre for Organismal Studies, University of Heidelberg, Heidelberg, Germany

*corresponding author (Email: klaus.harter@zmbp.uni-tuebingen.de)

\section{Abstract}

Protein-protein interaction studies provide valuable insights into cellular signaling. The well-studied brassinosteroid (BR) signaling is initiated by the hormone-binding receptor Brassinosteroid Insensitive 1 (BRI1) and its co-receptor BRI1 Associated Kinase 1 (BAK1). BRI1 and BAK1 were shown to interact independently with the Receptor-Like Protein 44 (RLP44), which is implicated in BRI1/BAK1-dependent cell wall integrity perception. To demonstrate the proposed complex formation of BRI1, BAK1 and RLP44, we established three-fluorophore intensity-based spectral Förster resonance energy transfer (FRET) and FRET-fluorescence lifetime imaging microscopy (FLIM) for living plant cells. Our evidence indicates that RLP44, BRI1 and BAK1 form a ternary complex in a distinct plasma membrane nanodomain. In contrast, although the immune receptor Flagellin Sensing 2 (FLS2) also forms a heteromer with BAK1, the FLS2/BAK1 complexes are localized in other nanodomains. In general, our three-fluorophore FRET approaches provide a feasible basis for studying the interaction and sub-compartmentalization of proteins in great detail. 


\section{Introduction}

Integration of different signaling cues at the cellular level is essential for the survival of any organism. In plants, for instance, mechanical damage to the cell wall causes attenuation of cellular growth response while resources are redistributed to repair processes. With the advent of high- and super-resolution microscopy techniques, the discovery of protein localization in nanodomains, the spatial organization of receptors and accompanying proteins (e.g. co-receptors) in the plasma membrane (PM) had come into focus. This has prompted new questions regarding, for example, the extent to which the constituents of a given signaling complex or nanodomain are restricted to it or disintegrate upon signal perception.

A well-understood pathway in plants is the brassinosteroid (BR) hormone signal transduction, mediated by the PM-resident receptor kinase BRI1 and its co-receptor BAK1. The binding of BR to BRI1's extracellular domain increases its interaction with BAK1 and the re-arrangement of proteins in the complex (review as reference), eventually leading to the auto- and trans-phosphorylation of their Ser/Thr-kinase domains ${ }^{1,2}$. These PM-resident events result in the differential regulation of BRresponsive genes by a nucleo-cytoplasmic signaling cascade ${ }^{3-6}$ and the activation of PM-resident P-type proton pumps ${ }^{7,8}$.

RLP44 was previously described to play a role in cell wall integrity sensing via modulation of BRI1/BAK1-dependent signaling ${ }^{9}$ and to interact with BRI1 and BAK1 ${ }^{9,10}$. This suggests that RLP44 directly affects the activity of the BRI1/BAK1 complex upon input from the cell wall, as a scaffold protein for the establishment of a specific BRI1/BAK1 complex in the PM ${ }^{2}$.

To test the hypothesis of ternary RLP44/BRI1/BAK1 complex formation, we at first had to establish three-fluorophore, intensity-based spectral FRET and FRET-FLIM in plant cells. FRET is the non-radiative energy transfer from a donor to an acceptor fluorophore by dipole-dipole interaction, which is only possible over small distances and depends on the relative dipole moment orientation of the donor and acceptor fluorophores ${ }^{11}$. FRET manifests itself by an alteration of the donor and acceptor fluorescence intensities, but also decreases the donor's excited state lifetime due to the additional relaxation path from the donor to the acceptor ${ }^{12}$. In contrast to twofluorophore FRET, an intermediate acceptor fluorophore is included in the energy transfer pathway of three-fluorophore FRET. As the plant cell wall cannot be 
penetrated by organic dyes, our approach depends solely on genetically encoded fluorophores. Here, we exemplary choose monomeric Turquoise 2 (mTRQ2) as donor, monomeric Venus ( $\mathrm{mVEN}$ ) as first acceptor and monomeric red fluorescence protein 1 (mRFP) as second acceptor.

Based on theoretical calculations we experimentally show by intensity-based spectral FRET and FRET-FLIM that RLP44, BRI1 and BAK1 are unified in a ternary complex that likely forms in a distinct nanodomain in the PM, which is spatially clearly distinct from the FLS2/BAK1 complex-containing nanodomain. In addition, we propose FRETFLIM to be always the better choice for the analysis of ternary protein complex formation and relative proximity estimates in plant cells. 


\section{Results}

\section{Physicochemical properties of the used fluorophores}

The selected fluorophores substantially influence the quality of the FRET data. All of our genetically encoded fluorophores were monomeric, minimizing false-positive FRET originating from aggregation. MTRQ2 was used as donor, as it has numerous advantages including a long, mono-exponential fluorescence lifetime (FLT) ${ }^{13}$. The spectral overlap of $\mathrm{mTRQ} 2$ emission with the absorbance of the first acceptor mVEN is high, yielding a large Förster distance $R_{0}{ }^{14-16}$. The second acceptor is mRFP, which has a large spectral overlap with the first acceptor (mVEN). The maturation time of the fluorophores is also a crucial factor for the FRET efficiency (ERET) ${ }^{17,18}$. In our threefluorophore system, both mVEN and mRFP have faster maturation times than mTRQ2, ensuring favorable FRET conditions (more details about the fluorophores in SI Table 1).

\section{Calculated properties of the used three-fluorophore FRET system}

To determine the operational FRET range for the chosen fluorophores, the corresponding Förster distances $\left(\mathrm{R}_{0}\right)$ were calculated (Table 1 ) as well as the distance corresponding to $10 \%$ FRET efficiency $\left(\mathrm{r}_{\mathrm{E}=10 \%)}\right.$. This last parameter best illustrates the measurement limit for FRET that can be realized with standard FRET microscopy measurements. For the complex energy flow in a three-fluorophore setting, $\mathrm{r}_{\mathrm{E}=10 \%}$ was calculated for the mTRQ2/mRFP pair, adding mVEN as intermediate acceptor. Especially in the case of receptor complex nanodomains in the PM, higher complex stoichiometries are expected. Additionally, in such a two-dimensional space it is more likely to have spatial situations where a donor is in range of several acceptors ${ }^{19}$. Therefore, we also calculated how this effect might influence the energy transfer. According to the absorption and emission spectra of the used fluorophores (Fig. 1a), the emission of mTRQ2 shows a significant spectral overlap with the absorption spectra of both mVEN and mRFP (Fig. 1a). Furthermore, the emission spectrum of mVEN substantially overlaps with the absorption spectrum of mRFP, enabling energy transfer from mTRQ2 to mVEN, mVEN to mRFP and mTRQ2 to mRFP. Ro was calculated with $\mathrm{n}=1.4$ and $\mathrm{K}^{2}=2 / 3$ according to ${ }^{20}$, using the spectra and photophysical data from the original publications (SI Table 2). The resulting Förster distances Ro were $5.7 \mathrm{~nm}$ for the mTRQ2/mVEN, $5.2 \mathrm{~nm}$ for the $\mathrm{mVEN} / \mathrm{mRFP}$ and 5.1 
$\mathrm{nm}$ for the mTRQ2/mRFP pair (Table 1, Fig. 1b). Evidently, the same trend is observed for $r_{E=10 \%}$, which is highest for the mTRQ2/mVEN pair and lowest for the mTRQ2/mRFP pair (Table 1, Fig. 1b). In addition, FRET was calculated not to be limited to sequential energy transfer from mTRQ2 via mVEN to mRFP but also from mTRQ2 to mRFP directly (Table 1, Fig. 1c).
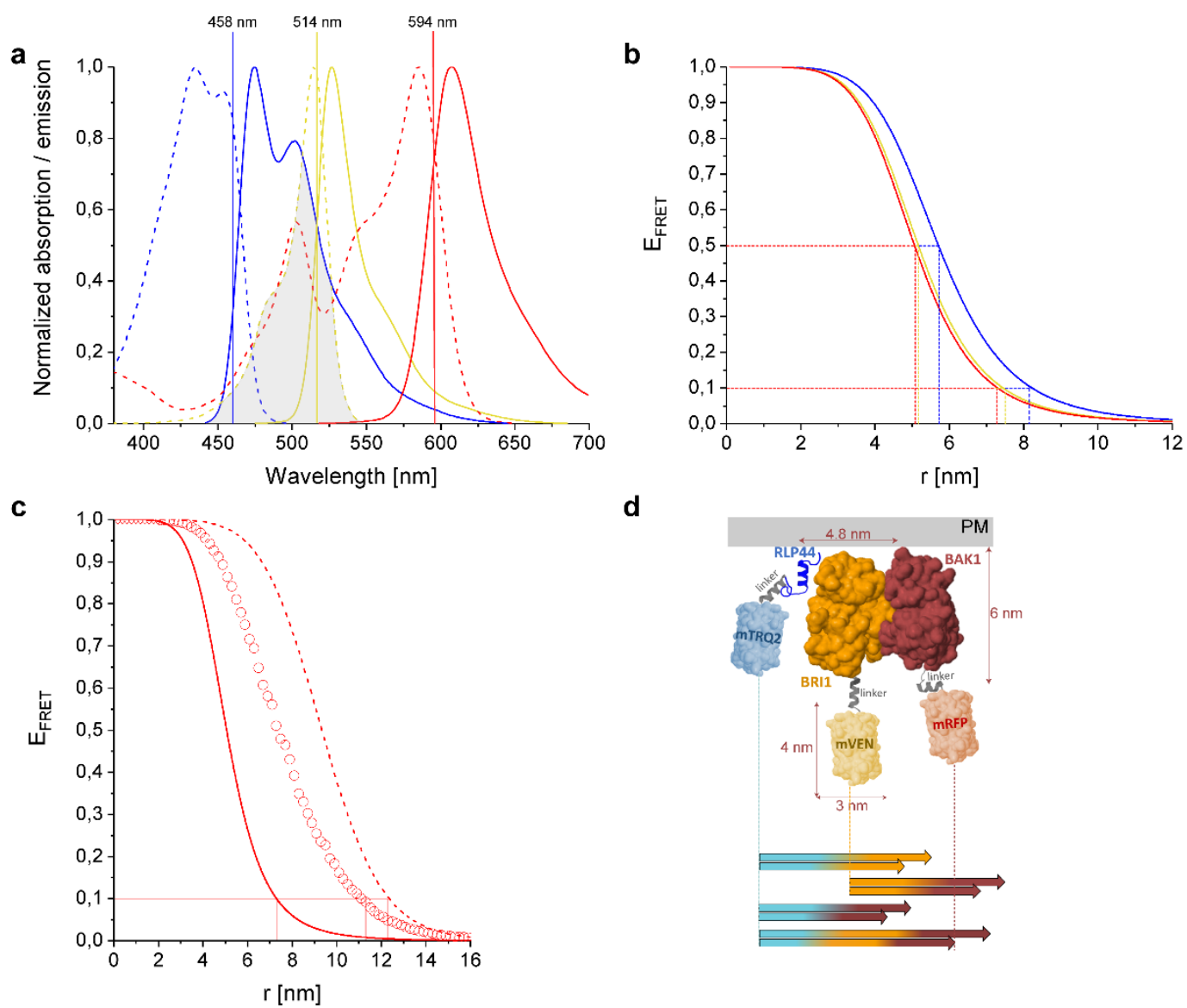

d

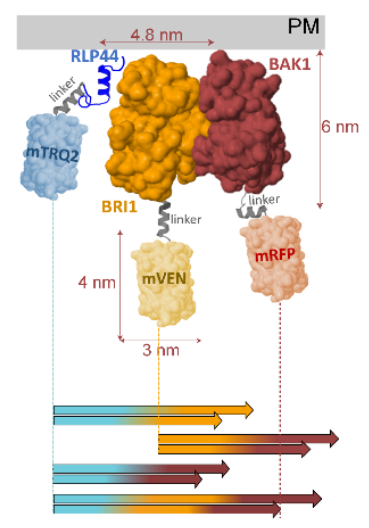

Fig. 1 Spectroscopic and FRET properties of the used fluorophores and dimensions of the analyzed RLP44-mTRQ2, BAK1-mVEN and BRI1-mRFP fusion proteins. $a$, Normalized absorption (dotted lines) and emission (solid lines) spectra of mTRQ2 (blue), mVEN (yellow) and mRFP (red). mTRQ2 is donor to both mVEN as acceptor 1 as well as mRFP as acceptor 2; mVEN is also donor to mRFP. The shaded area corresponds to the spectral overlap between donor emission and acceptor absorption, shown here exemplary for the mTRQ2/mVEN FRET pair. The laser lines for excitation of mTRQ2 (blue), mVEN (yellow) and mRFP (red) are marked as vertical lines at their respective wavelength positions. b, FRET efficiencies ( $E_{F R E T}$ ) for the distances $(r)$ between donor and acceptor fluorophores for mTRQ2mVEN (blue), mVEN-mRFP (yellow) and mTRQ2-mRFP (red). The Förster distances $R_{0}$ at $E_{F R E T}=50 \%$ as well as the distances that correspond to $E_{F R E T}=10 \%\left(r_{E=10 \%}\right)$ for each pair 
are marked with dashed lines in the respective color. c, $E_{F R E T}$ in dependence of distance $r$ between mTRQ2 and mRFP without (solid line) and with intermediate mVEN located either equidistantly (dashed line) or variably (circles) between mTRQ2 and mRFP. For the latter, mVEN was placed at 1,000 random positions for each mTRQ2/mRFP distance, averaging over the resulting $\mathrm{E}_{F R E T}$ values. The $\mathrm{r}_{\mathrm{E}=10 \%}$ distances are marked with red lines. $\mathbf{d}$, Composite image of the cytoplasmic domains of RLP44 (blue), BRI1 (orange) and BAK1 (brown) fused to the 15 amino acid-long Gateway®-linker to either mTRQ2 (light blue), mVEN (light yellow) or mRFP (light red). The structures of the cytoplasmic domains of BAK1, BRI1 and the fluorophores are shown as solvent-accessible surface models, while the model structures of the cytoplasmic domain of RLP44 and the three Gateway®-linkers (grey) were predicted with PEP-FOLD3 and depicted as cartoons. The orientation of the kinase domains of BRI1 and BAK1 to each other was designed according to a molecular docking analysis in orientation to the PM. ${ }^{27}$ The colored arrows below the structures show the $r_{E=10 \%}$ distances for all FRET pairs and the three-chromophore FRET cascade with mVEN inserted at random positions, calculated for a donor to acceptor complex ratio of 1:1 (lower arrows) and 1:2 (upper arrows). The precise values for $b, c$ and $d$ are listed in Table 1.

For large distances between mTRQ2 and mRFP no direct FRET is expected to be possible. Therefore, the introduction of the intermediate acceptor mVEN could increase the dynamic range between mTRQ2 and mRFP. To estimate this long-range effect, we investigated how the presence of mVEN affects the energy transfer to mRFP in such a FRET cascade ${ }^{21}$ and calculated an increase for $\mathrm{r}_{E=10 \%}$ from $8.2 \mathrm{~nm}$ to $12.4 \mathrm{~nm}$ (Fig. 1c, Table 1), which is in agreement with the increase in effective FRET distance reported previously ${ }^{22}$. However, the authors of 28 fixed the fluorophore positions along a DNA helix with equidistant separation between the fluorophores. In multimeric protein complexes, however, neither the complex geometry nor the exact position of the attached fluorophores are usually known. Therefore, we additionally calculated an averaged $\mathrm{r}_{\mathrm{E}=10 \%}$ value when $\mathrm{mVEN}$ is inserted at random positions between mTRQ2 and mRFP. In this more realistic representation of our dynamic plant system, we found a slightly smaller increase of $\mathrm{r}_{\mathrm{E}=10 \%}$ from $8.2 \mathrm{~nm}$ to $11.1 \mathrm{~nm}$ (Fig. 1c, Table 1). This is by far large enough to be able to detect long-range interactions with our three-fluorophore FRET approach.

In case of higher complex stoichiometries, the $\mathrm{r}_{\mathrm{E}=10 \%}$ values increase even further. Due to the so-called FRET "antenna" effect the distance term in the ERET calculation changes to $n^{-1} r^{6}$, where $n$ is the number of identical and equidistant acceptors per 
donor ${ }^{19}$. Additionally, the orientation factor $\mathrm{K}^{2}$, which is usually assumed to be $2 / 3$ for freely rotating molecules, increases due to selection bias for multiple acceptors ${ }^{19}$ (known as the FRET "surplus" effect ${ }^{23}$ ) leading to an increase of Ro and thus EFRET. Therefore, when mVEN is inserted in the middle or at a randomized position in the FRET arrangement, even the cautious assumption of at least two acceptors in the range of each donor results in an increase of $r_{10 \%}$ from 12.4 to $14.6 \mathrm{~nm}$ and 11.1 to $13.1 \mathrm{~nm}$, respectively (Table 1, Supplementary Information).

In summary, depending on the donor-to-acceptor ratio and on the basis of a variable arrangement, an energy transfer from mTRQ2 via mVEN to mRFP could occur at distances up to $13.1 \mathrm{~nm}$. Hence, long-range ternary protein interactions are verifiable by fusing mTRQ2, mVEN and mRFP to proteins of interest.

Table 1: FRET combinations ( $1^{\text {st }}$ column), their Förster distances $\left(\mathrm{R}_{0}\right)\left(2^{\text {nd }}\right.$ column) and fluorophore distances that correspond to $10 \%$ FRET efficiency $\left(r_{10} \%\right)$, calculated for a linear arrangement with either one ( $3^{\text {rd }}$ column) or two acceptors ( $4^{\text {th }}$ column) in range of each corresponding donor (see also SI Table 2).

\begin{tabular}{|l|c|c|c|}
\hline FRET combinations & $R_{0}[\mathrm{~nm}]$ & $\begin{array}{c}r_{10 \%}[\mathrm{~nm}], \\
\mathrm{D}: \mathrm{A}=1: 1\end{array}$ & $\begin{array}{c}\mathrm{r}_{10 \%}[\mathrm{~nm}], \\
\mathrm{D}: \mathrm{A}=1: 2\end{array}$ \\
\hline mTRQ2-mVEN & 5.7 & 8.2 & 9.7 \\
\hline mVEN-mRFP & 5.2 & 7.4 & 8.8 \\
\hline mTRQ2-mRFP & 5.1 & 7.3 & 8.6 \\
\hline mTRQ2-mVEN-mRFP (middle position) & - & 12.4 & 14.6 \\
\hline mTRQ2-mVEN-mRFP (random position) & - & 11.1 & 13.1 \\
\hline
\end{tabular}

\section{Structural simulation of the arrangement of the fluorophore-tagged proteins for the estimation of the FRET range}

To assess how the maximum dynamic range of about $13.1 \mathrm{~nm}$ relates to the size of our proteins of interest, we arranged the cytoplasmic domains of RLP44, BRI1 and BAK1 fused to the respective fluorophores and the calculated $r_{E=10 \%}$ values between mTRQ2, mVEN and mRFP in scale in a graph (Fig. 1d). As the structures of the fluorophores and the cytoplasmic domains of BRI1 and BAK1 are available, a solventaccessible surface representation was generated to estimate the protein sizes ${ }^{24-26}$. The orientation of the BRI1 and BAK1 kinase domains to each other was depicted according to the highest probability after a molecular docking analysis ${ }^{27}$. The structures of RLP44's cytoplasmic domain and the Gateway®-linkers were not available and predicted with PEP-FOLD3 ${ }^{28}$ (for details see Methods). The 
stoichiometry-adjusted $\mathrm{r}_{\mathrm{E}=10 \%}$ values for all FRET pairs are between 8.6 and $9.7 \mathrm{~nm}$ (Table 1) and, therefore, could span the distance of two kinase domains, which have diameters of about $4.8 \mathrm{~nm}$ (Fig. 1d). Most importantly, while RLP44-mTRQ2 and BAK1-mRFP are too far apart for FRET in this exemplary linear arrangement, threefluorophore FRET from RLP44-mTRQ2 via BRI1-mVEN to BAK1-mRFP is able to span this distance (Fig. 1e) The same is expected for a RLP44-mTRQ2/BAK1mVEN/BRI1-mRFP arrangement.

\section{Calculation of cross-excitation and bleed-through in intensity-based spectral FRET measurements}

The vast majority of investigations in animal cells have assessed three-fluorophore FRET with intensity-based methods, specifically by quantitative acquisition of spectra, using predominantly organic dyes. Therefore, we decided to first assess the ternary complex formation for the selected fusion proteins in plant cells with this method.

Regardless of the stoichiometry of the complexes, the expression levels of the fluorophore-fused proteins have a large influence on the spectra of intensity-based FRET, due to cross-excitation and bleed-through. This is demonstrated in the simulated emission spectra of Fig. 2, calculated for a complex stoichiometry of 1:1:1 and a linear arrangement of mTRQ2, mVEN and mRFP placing mVEN at equal distance to the other two fluorophores. With this fluorophore ratio, inter-fluorophore distances above $10 \mathrm{~nm}$ (no FRET) and an excitation wavelength of $458 \mathrm{~nm}$, an intensity peak at around $525 \mathrm{~nm}$ was calculated to appear due to cross-excitation (Fig. 2a, top). With inter-fluorophore distances of $7 \mathrm{~nm}$ (Fig. 2a, middle) and $5 \mathrm{~nm}$ (Fig. 2a, bottom), the energy transfer from mTRQ2 to mVEN and mRFP became apparent in the simulated spectra, as the relative mTRQ2 intensity peak decreased, while the mVEN and mRFP peaks increased. The theoretical bleed-through and crossexcitation depend strongly on the donor-to-acceptor ratio of the fluorophores (Fig. $2 b$ and Supplementary Information). Importantly, the bleed-through and cross-excitation effects have a larger influence on the apparent mVEN signal than FRET itself: Already a ratio of $1: 2$ for $m T R Q 2 / m V E N$ results in a peak of the fluorescence intensity at around $525 \mathrm{~nm}$ similar to that calculated for an inter-fluorophore distance of $7 \mathrm{~nm}$ (compare Fig. 2a, middle and Fig. 2b, top). However, the different mRFP intensity progression was well observable (compare Fig. $2 \mathrm{a}$ with $2 \mathrm{~b}$. This underscores the 
necessity for a careful calibration of the fusion protein amounts. Therefore, the bleedthrough and cross-excitation are always quantified in the presented absorption and emission spectra as well as in the confocal images (Supplementary Information).

a
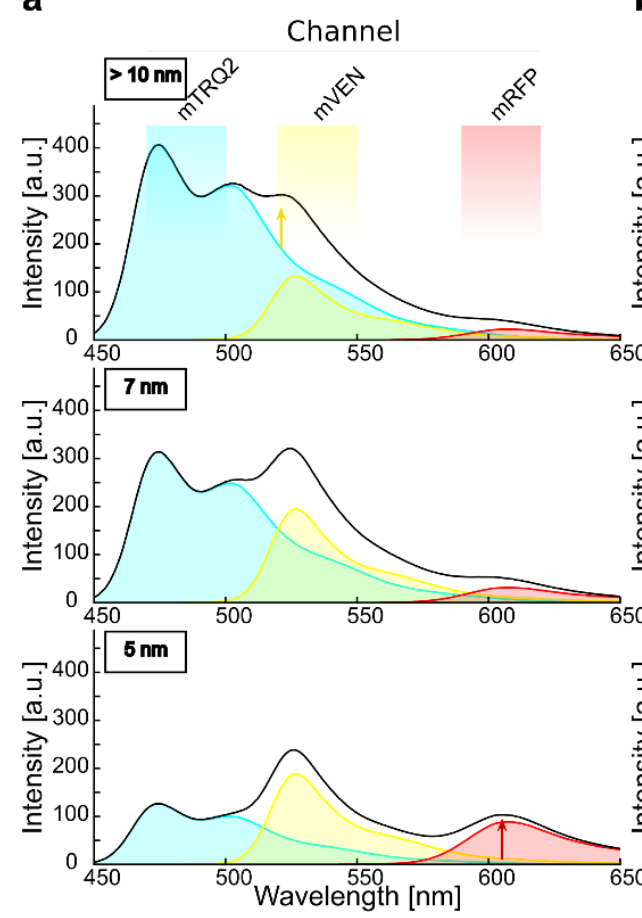

b
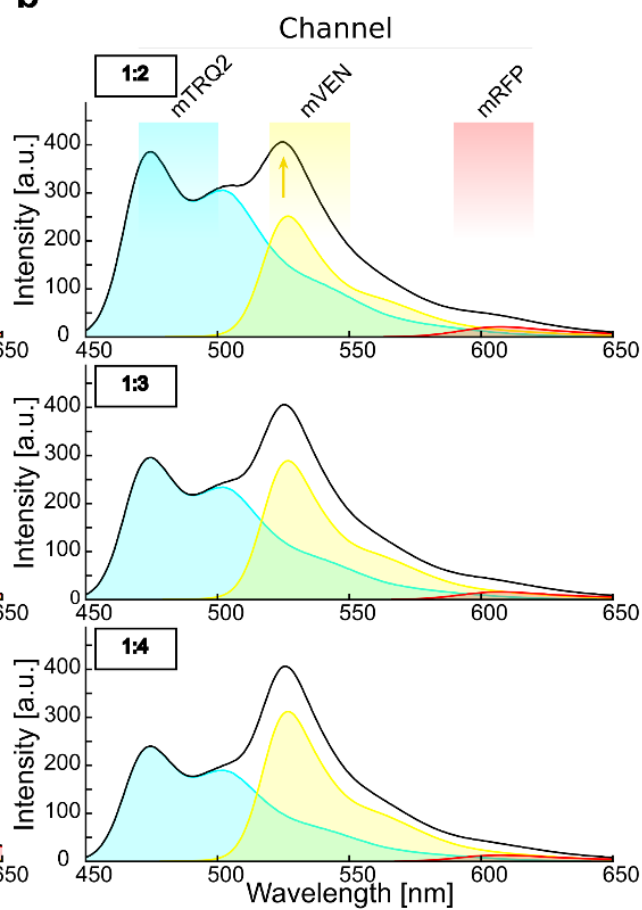

Fig. 2 | Simulation of emission spectra reveals the crucial impact of relative fluorophore quantities for intensity-based spectral FRET in a three-chromophore arrangement. a, Simulation of the spectra that result from excitation of mTRQ2 with $458 \mathrm{~nm}$ for a mTRQ2, mVEN and mRFP stoichiometry of 1:1:1 and a linear arrangement of the three fluorophores, while placing $\mathrm{mVEN}$ at equal distance to the other two fluorophores. The emission spectra of mTRQ (blue area), mVEN (yellow area) and mRFP (red area) and their combined spectrum (black line) are calculated for equal fluorophore expression levels. The respective emission detector channels are depicted above the plots. With inter-fluorophore distances larger than $10 \mathrm{~nm}$, FRET is negligible (top), but cross-excitation of mVEN (yellow arrow) is apparent. For inter-fluorophore distances of $7 \mathrm{~nm}$ (middle) and $5 \mathrm{~nm}$ (bottom), FRET lowers the emission intensity of mTRQ2, while the intensity peaks of mVEN at about $525 \mathrm{~nm}$ and of mRFP at about $610 \mathrm{~nm}$ (red arrow) increase. b. Spectra simulation as in $\mathbf{a}$, but without consideration of FRET, calculated for different mTRQ2/mVEN fluorophore ratios. With rising mTRQ2/mVEN ratios of 1:2 (top), 1:3 (middle) and 1:4 (bottom), the intensity peak of mVEN (yellow arrow) increases. Thus, a mTRQ2/mVEN ratio of 1:2 (b, top) results in a similar spectral shape than with equimolar fluorophores subjected to FRET at a distance of $7 \mathrm{~nm}$ (a, middle). 


\section{Experimental determination of cross-excitation and bleed-through in plant cells}

It was previously shown that BRI1/BAK1, RLP44/BRI1 and RLP44/BAK1 form heteromers in plant cells ${ }^{9,10,29}$. In contrast, the PM-localized immune response mediating Flagellin Sensing 2 (FLS2) receptor does not interact with RLP44 ${ }^{30,31}$ and was selected as a negative control in the further analyses. To perform our study, the spectra were acquired in transiently transformed Nicotiana benthamiana epidermal leaf cells. All fusion proteins localized to the PM (SI Fig. 1).

The fluorescence emission spectra of the three fusion constructs (RLP44-mTRQ2, FLS2-mVEN and BAK1-mRFP) were recorded in separate experiments after excitation with light of $458 \mathrm{~nm}$, with only FLS2-mVEN showing a significant amount of cross-excitation (Fig. 3a). The quantification of the fluorescence emission of RLP44mTRQ2 after excitation at $458 \mathrm{~nm}$ is a measure for its protein level, manifesting an average intensity of 120 arbitrary units (a.u.) (Fig. 3b). It was reflected in an emission peak intensity of 120 a.u. in the spectrum (Fig. 3a). A relative high accumulation level of FLS2-mVEN with an average intensity of 200 a.u. after excitation at $514 \mathrm{~nm}$ (Fig. 3b) caused a much smaller background signal of 8 a.u. in the spectrum after excitation with light of $458 \mathrm{~nm}$ (Fig. 3a). The excitation of BAK1-mRFP with light of $561 \mathrm{~nm}$ gave an average intensity of 110 a.u. (Fig. 3b) but did not result in a distinct peak in the emission spectrum after excitation with light of $458 \mathrm{~nm}$ (Fig. 3a). Thus, the different accumulation levels of the fusion proteins led to the same shape of the fluorescence emission spectra but with variations in the peak intensities (SI Fig. 2).

\section{Intensity-based FRET analysis of dual protein-protein interactions}

First, we assessed FRET from the mTRQ2 to the mVEN fusion proteins. Since our simulations indicate that the amount of the fusion proteins has a larger influence on the shape of the spectra than FRET itself, a correction for the relative proteins levels was imperative. Therefore, it was always necessary to compare the spectra with the same donor-to-acceptor ratios of RLP44-mTRQ2/BRI1-mVEN with that of RLP44mTRQ2/FLS2-mVEN. To this end, each recorded spectrum was subjected to spectral unmixing to determine the relative proportions of mTRQ2 and mVEN (Supplementary Information). Then, the spectral unmixing information was combined with the respective estimates of fusion protein levels. To do so, each channel was imaged with 
sequential excitation before acquisition of the spectra (excitation with only $458 \mathrm{~nm}$, then only $514 \mathrm{~nm}$, lastly only $561 \mathrm{~nm})$.

a

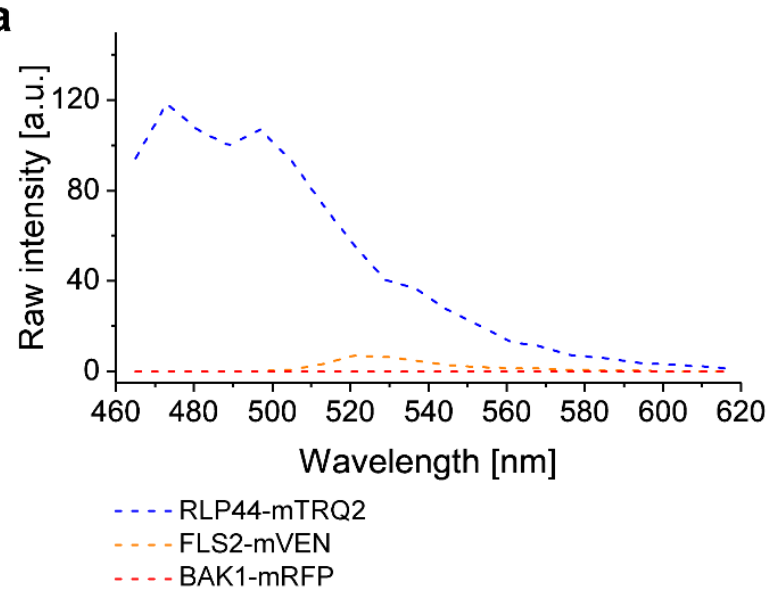

C

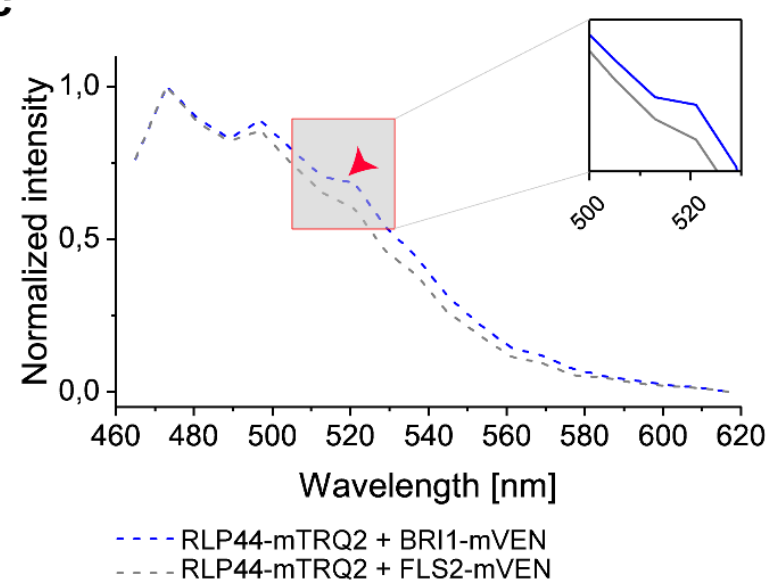

e

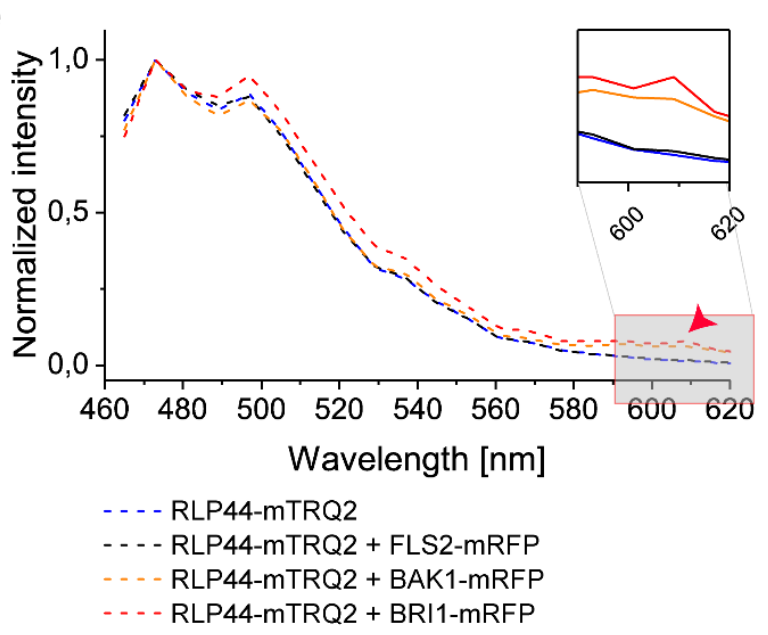

b

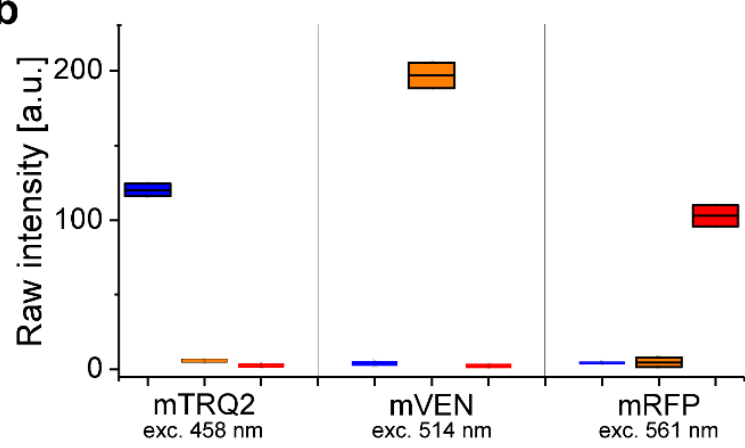

RLP44-mTRQ2

FLS2-mVEN BAK1-mRFP
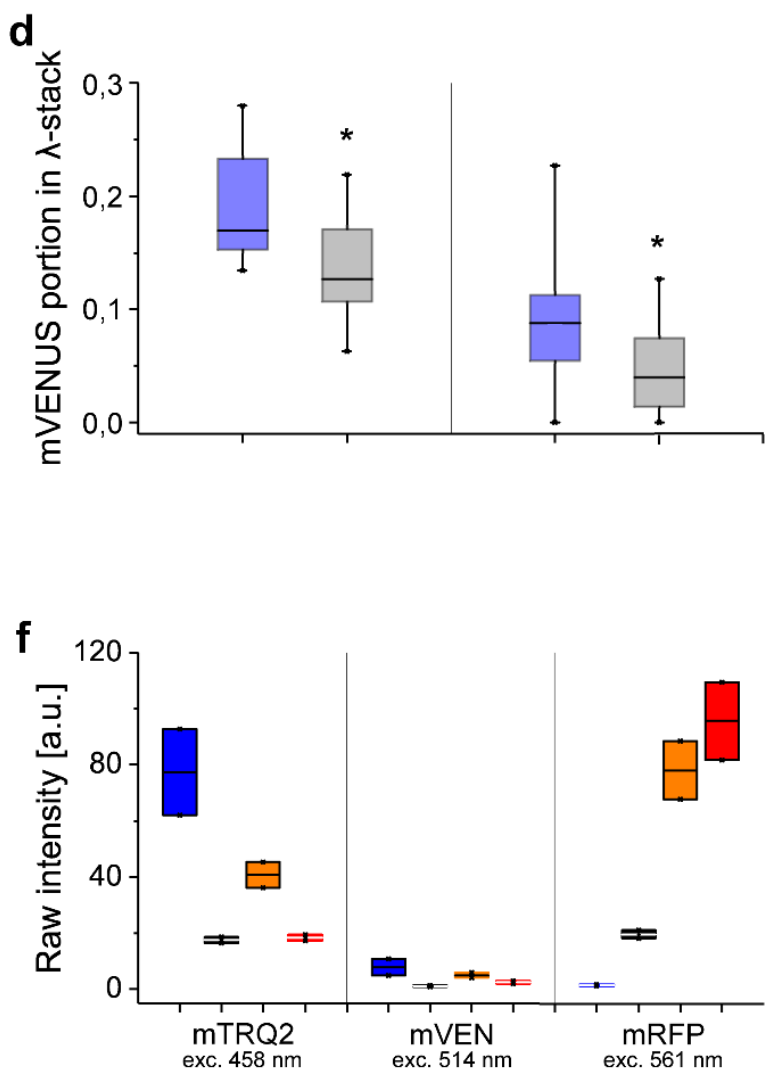

RLP44-mTRQ2

RLP44-mTRQ2 + FLS2-mRFP

RLP44-mTRQ2 + BAK1-mRFP

RLP44-mTRQ2 + BRI1-mRFP

Fig. 3 | Interaction of RLP44 with BAK1 and BRI1 is detectable in the plasma membrane of $N$. benthamiana epidermal leaf cells by intensity-based spectral FRET. a, Wavelengthdependent intensity of fluorescence emission after irradiation of the cells with $458 \mathrm{~nm}$ light for RLP44-mTRQ2 (blue), FLS2-mVEN (orange) and BAK1-mRFP (red). b, Emission intensity after irradiation of the cells with light of different wavelengths in the mTRQ2 channel (left, 
$458 \mathrm{~nm}$ ), mVEN channel (middle, $514 \mathrm{~nm}$ ) and mRFP channel (right, $561 \mathrm{~nm}$ ) of 8-bit images for RLP44-mTRQ2 (blue), FLS2-mVEN (orange) and BAK1-mRFP (red) after correction for spectral bleed-through. c, Wavelength-dependent normalized fluorescence emission for the co-expression of RLP44-mTRQ2 with BRI1-mVEN (blue) or with FLS2-mVEN (grey). For both spectra, the average donor-to-acceptor ratio was around 1:4 (0.26). The FRET-relevant wavelength area is highlighted in an enlarged section and the occurrence of FRET from RLP44-mTRQ2 to BRI1-mVEN is indicated by a red arrow head. The data are presented as mean ( $n \geq 13)$. d, Protein amount-dependent, relative mVEN emission signal in the fluorescence spectra after irradiation of the cells with light of $458 \mathrm{~nm}$ light. Fusion protein ratios of about 1:4 (left) and 1:10 (right) of the RLP44-mTRQ2/BRI1-mVEN pair (blue) and RLP44TRQ2/FLS2-mVEN pair (grey) are shown. Significant changes [ $=7$ (left), $n=12$ (right)] in a two-sided 2-sample t-test with $p<0.05$ are indicated by asterisks. e, Wavelength-dependent normalized fluorescence emission after irradiation of the cells with light of $458 \mathrm{~nm}$ for RLP44mTRQ2 alone (blue), RLP44-mTRQ/FLS2-mRFP (black), RLP44-mTRQ/BAK1-mRFP (orange) and RLP44-mTRQ/BRI1-mRFP (red). The FRET-relevant wavelength area is highlighted in an enlarged section and the occurrence of FRET fromRLP44-mTRQ2 to BAK1mRFP or BRI1-mRFP is indicated by a red arrow head. $\mathbf{f}$, Emission intensity after irradiation of the cells with light of different wavelength in the mTRQ2 (left), mVEN (middle) and mRFP channel (right) of 8-bit images for of RLP44-mTRQ2 (blue), RLP44-mTRQ/FLS2-mRFP (black), RLP44-mTRQ/BAK1-mRFP (orange) and RLP44-mTRQ/BRI1-mRFP (red). For further details see $\mathbf{b}$. For the statistical evaluation see Methods. The boxplots in $\mathbf{b}$ and $\mathbf{f}$ represent the measured data with the average (here equivalent to the median) as a solid black line within the box that is restricted by $+/$ - standard deviation. The boxplot in $\mathbf{d}$ represents all data with the median as a solid black line within the box that is restricted by the first quartile (25\%; lower end) and the third quartile (75\%; upper end). Whiskers show the minimum and maximum of the measurements, respectively

In the spectra with an identical donor-to-acceptor sample ratio, the peak intensity value at about $525 \mathrm{~nm}$ was higher for the RLP44-mTRQ2/BRI1-mVEN than for the RLP44mTRQ2/FLS2-mVEN sample (Fig. 3c). The integration of the spectral unmixing results and donor-to-acceptor ratios revealed that the proportion of mVEN emission in the spectra of the RLP44-mTRQ2/BRI1-mVEN sample differed significantly from that of the RLP44-mTRQ2/FLS2-mVEN sample (Fig. 3d). This was also true for donor-toacceptor ratios of approximately $1: 10$ and 1:4 (Fig. 3d). In conclusion, FRET from RLP44-mTRQ2 to BRI1-mVEN was observed, but only after careful comparison with the data recorded for the non-FRET RLP44-mTRQ2/FLS2-mVEN control pair. 
We next tested, whether an energy transfer from the mTRQ2 to mRFP fusion proteins is detectable. For the co-accumulation of RLP44-mTRQ2 with FLS2-mRFP, no significant increase of the emission at around $610 \mathrm{~nm}$ was detected compared to the donor alone control (Fig. 3e). In contrast, co-accumulation of RLP44-mTRQ2 with BAK1-mRFP or BRI1-mRFP led to a significant increase of the emission at around $610 \mathrm{~nm}$ compared to the control (Fig. 3e). The ratio in fluorescence intensity between mTRQ2 and mRFP was 1:1 for the RLP44-mTRQ2/FLS2-mRFP pair (Fig. 3f), whereas RLP44-mTRQ2/BRI1-mRFP and RLP44-mTRQ2/BAK1-mRFP were 1:2 and 1:5, thus higher than in the RLP44-mTRQ2/FLS2-mRFP control (Fig. 3f). After correction for the different fusion protein amounts, spectrally detectable FRET clearly occurred from RLP44-mTRQ2 to BAK1-mRFP or to BRI1-mRFP but not to FLS2mRFP.

\section{Intensity-based FRET analysis of ternary protein complex formation}

When RLP44, BRI1 and BAK1 form a ternary protein complex, the average distance between RLP44 and BAK1 might be altered by the presence of BRI1. For example, BRI1 may be located between RLP44 and BAK1, increasing the distance between them. Also the total number of RLP44/BAK1 pairs may be reduced, as additional pairs such as RLP44/BRI1 or BAK1/BRI1 can be formed ${ }^{32}$.

To test this possibility, we expressed BRI1 with a non-fluorescent HA-tag (BRI1-HA) in tobacco cells (SI Fig. 3). Co-expression of BRI1-HA did not change the shape of the RLP44-mTRQ2 spectrum (compare Fig. 4a and Fig. 3a). As described above, coaccumulation of RLP44-mTRQ2 with BAK1-mRFP yielded a FRET-induced emission peak at around $610 \mathrm{~nm}$ (Fig. 4a). This peak was no longer present when BRI1-HA was co-expressed with RLP44-mTRQ2 and BAK1-mRFP (Fig. 4a). In contrast, the coexpression of RLP44-mTRQ2/FLS2-mVEN/BRI1-mRFP retained the intensity peak at around $610 \mathrm{~nm}$ (SI Fig. 4). Thus, the addition of BRI1-HA increased the average distance between RLP44-mTRQ2 and BAK1-mRFP or led to alterations in the fluorophore-tagged protein levels. As the latter was not the case (Fig. 4b), our results demonstrate that the distance between RLP44-mTRQ2 and BAK1-mRFP did indeed change upon BRI1-HA co-accumulation. When BRI1-mVEN was co-expressed instead of BRI1-HA, the emission peak at around $610 \mathrm{~nm}$ reappeared (Fig. 4c,d). At the same time, energy transfer from mVEN to mRFP was detected, as the emission 
peak of mVEN at $525 \mathrm{~nm}$ strongly decreased in the RLP44-mTRQ2/BRI1mVEN/BAK1-mRFP sample in comparison with the RLP44-mTRQ2/BRI1-mVEN sample which was not observed in the FLS2 control samples (Fig. 4a,c). Although the influence of cross-excitation of mVEN and subsequent FRET directly to mRFP could not be excluded entirely, this effect is negligible, as no energy transfer from mVEN to mRFP was observed for the RLP44/FLS2/FLS2 sample (SI Fig. 4). Likewise, the analysis of the different protein combinations did not change the donor-to-acceptor ratios significantly (Fig. 4d). In conclusion, the appearance of the emission peak at around $610 \mathrm{~nm}$, which was accompanied by the parallel reduction of the emission at around $525 \mathrm{~nm}$, fulfils the criteria for three-chromophore FRET from RLP44-mTRQ2 via BRI1-mVEN to BAK1-mRFP. In addition, the energy transfer described above was also observed when the acceptor fluorophores were exchanged (SI Fig. 4).

In conclusion, our data prove convincingly that FRET from mTRQ2 via mVEN to mRFP specifically occurred for the RLP44/BRI1/BAK1 and RLP44/BAK1/BRI1 combinations but not for the RLP44/BAK1/FLS2 combination. This indicates that RLP44, BRI1 and BAK1 form a specific ternary complex and/or are arranged in very close spatial proximity in the PM specifically. The successful establishment of intensity-based threefluorophore FRET now prompted us to test whether the complex formation could also be recorded by FLIM. 
a

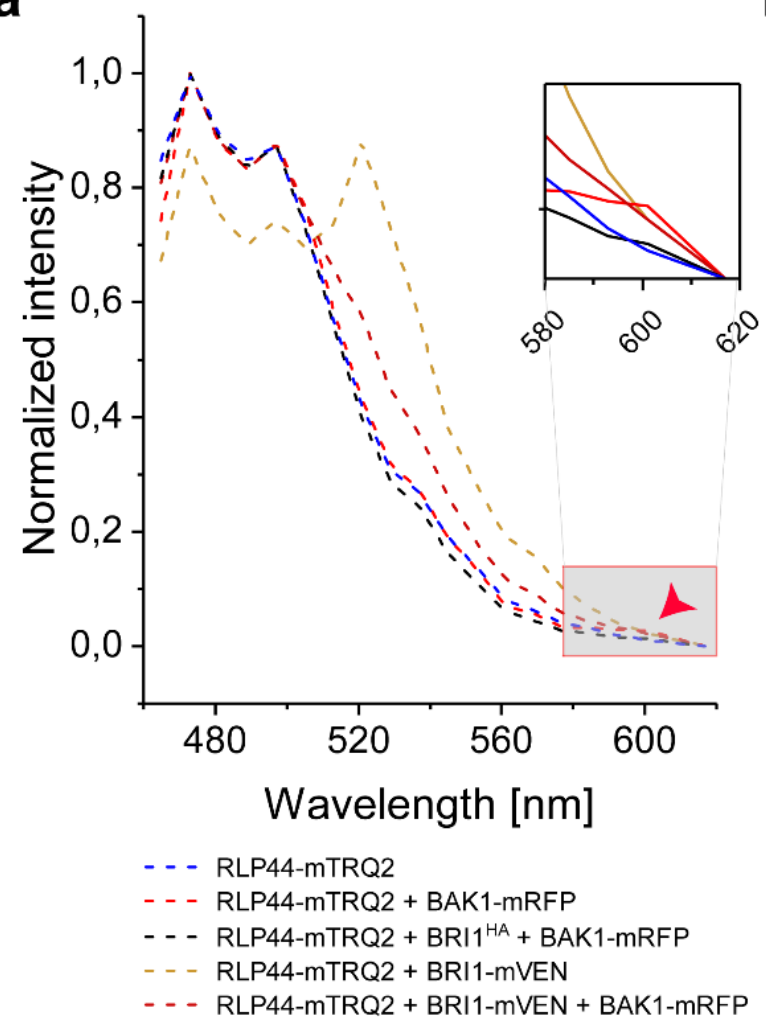

b

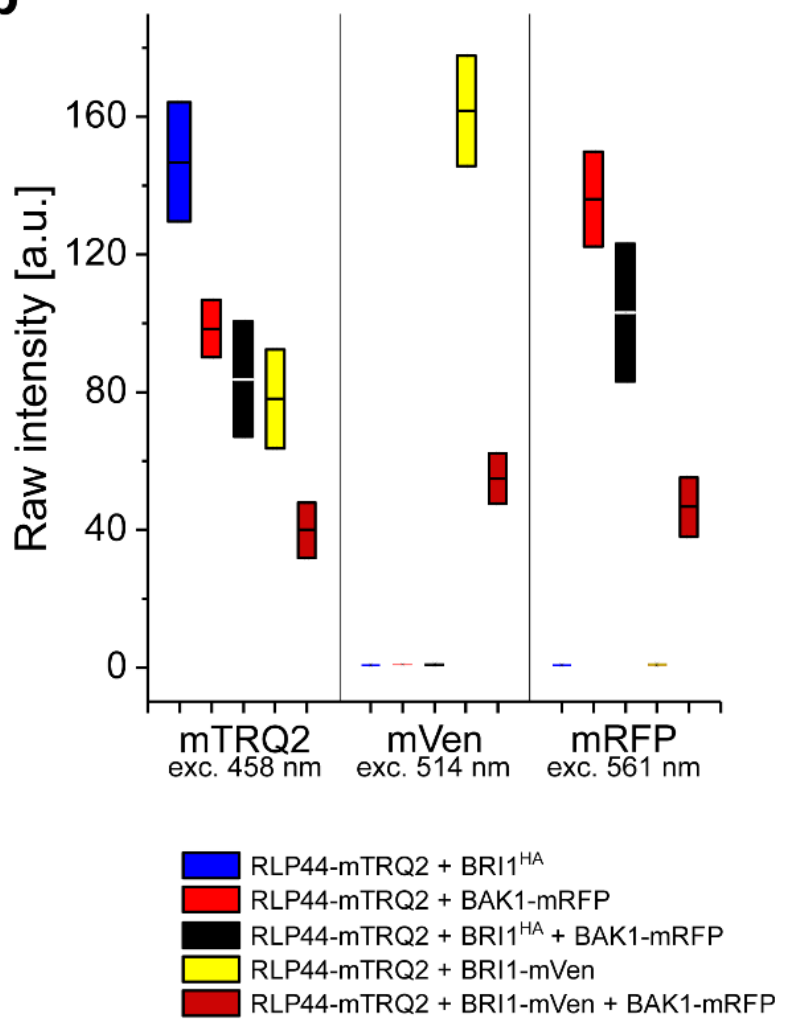

C

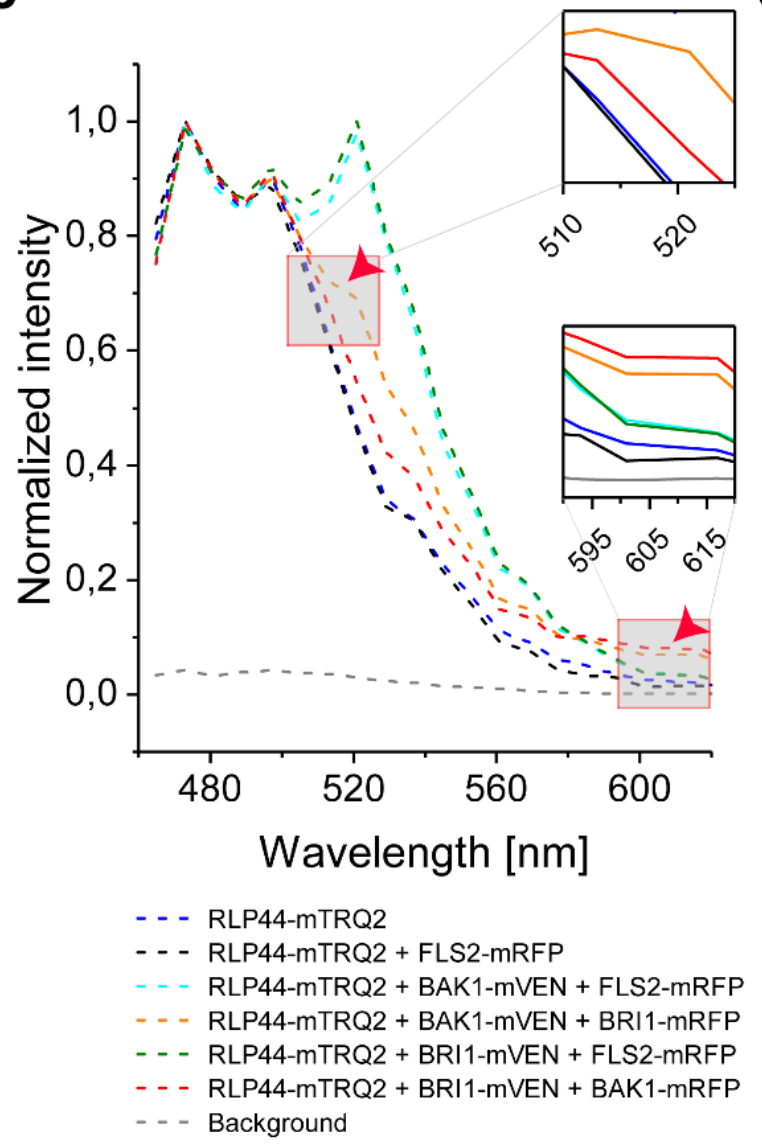

d

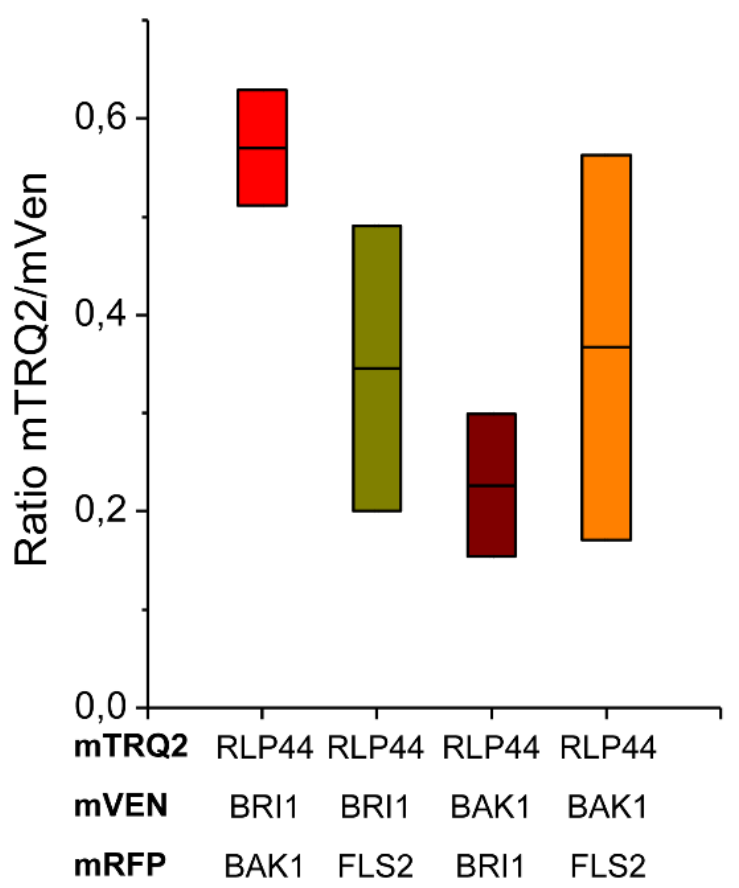


Fig. 4 | The formation of a ternary complex consisting of RLP44, BAK1 and BRI1 but not including FLS2 is detectable in the plasma membrane of $N$. benthamiana epidermal leaf by intensity-based spectral FRET. a, Wavelength-dependent normalized fluorescence emission after irradiation of the cells with $458 \mathrm{~nm}$ light for the expression of RLP44-mTRQ2 alone (blue) or co-expression with either BAK1-mRFP (light red) or HA-tagged BRI1 (BRI1 ${ }^{\mathrm{HA}}$ ) and BAK1-mRFP (black) or BRI1-mVEN (bronze) or BRI1-mVEN and BAK1-mRFP (dark red). The FRET-relevant wavelength area is highlighted in an enlarged section. The occurrence of FRET from mTRQ2 to mRFP is indicated by a red arrowhead. b, Emission intensity after irradiation of the cells with light of different wavelength in the mTRQ2 (left, $458 \mathrm{~nm}$ ), mVEN (middle, $514 \mathrm{~nm}$ ) and mRFP channel (right, $561 \mathrm{~nm}$ ) of 8-bit images for RLP44-mTRQ2 coexpressed with either BRI1 ${ }^{\mathrm{HA}}$ (blue), BAK1-mRFP (light red), BRI1 ${ }^{\mathrm{HA}}$ and BAK1-mRFP (black), BRI1-mVEN (yellow) or BRI1-mVEN and BAK1-mRFP (dark red). c, Wavelength-dependent normalized fluorescence emission after irradiation of the cells with $458 \mathrm{~nm}$ light for the expression of RLP44-mTRQ alone (blue) or co-expression with either BRI1-mVEN and BAK1mRFP (red) or BAK1-mVEN and BRI1-mRFP (orange) or BRI1-mVEN and FLS2-mRFP (green) or BAK1-mVEN and FLS2-mRFP (cyan) or with FLS2-mRFP (black). The FRETrelevant wavelength areas are highlighted in enlarged sections. The occurrence of FRET from mTRQ2 to mRFP at approximately $610 \mathrm{~nm}$ and the FRET-caused decrease of the mVEN signal at approximately $525 \mathrm{~nm}$ are indicated by red arrowheads. The background emission of the cells is shown as dashed grey line. $\mathbf{d}$, Fluorescence emission ratio after irradiation of the cells with light of different wavelength in the mTRQ2 channel (left), mVEN channel (middle) and mRFP channel (right) of 8-bit images for RLP44-mTRQ2 co-expressed with BRI1 ${ }^{\mathrm{HA}}$ and BAK1-mRFP (black), BRI1-mVEN (yellow) and BRI1-mVEN and BAK1-mRFP (dark red). See b for further details. The boxplots in $\mathbf{b}$ and $\mathbf{d}$ represent all data with the average (here equivalent to the median) as a solid black line within the box that is restricted by $+/$ - standard deviation. For the statistical evaluation see Methods.

\section{Measurement of in vivo RLP44/BRI1/BAK1 ternary complex formation by FRET-FLIM}

When studying three-way interactions with intensity-based spectral methods, the ratio between donor and acceptor molecules is of major importance, as spectral bleedthrough and cross-excitation mimics potential FRET. In contrast, when monitoring the fluorescence lifetime (FLT) of the donor by FLIM, no such careful and labor-intensive calibrations are required ${ }^{7,33}$.We therefore tested, whether the complex formation and 
spatial arrangement of RLP44, BRI1 and BAK1 can also be monitored and confirmed by changes in the FLT of MTRQ2.

We determined an average FLT of about 3.99 ns for RLP44-mTRQ2 (Fig 5a) as reported before ${ }^{13,15}$. Interestingly, a significant decrease in the FLT is observed for the co-accumulation of RLP44-mTRQ2 with BRI1 fused to either mVEN (3.54 ns) or mRFP (3.60 ns) or with BAK1-mRFP (3.54 ns), indicating FRET from RLP44-mTRQ2 to BRI1-mVEN, BRI1-mRFP or BAK1-mRFP (Fig. 5a). This constitutes the lower FLT limit that is expected when only heterodimers are present in the PM. Serving as a negative control, the co-expression of FLS2-mVEN (3.70 ns) or FLS2-mRFP (3.90 ns) with RLP44-mTRQ2 did not cause a significant decrease in mTRQ2's FLT (Fig. 5a,b). The co-accumulation of FLS-mRFP with RLP44-mTRQ2 and BRI1-mVEN did not further reduce the FLT of RLP44-mTRQ2 (3.60 ns) compared to the situation, where FLS2-mRFP is not present (Fig. 5a). In contrast, the presence of BAK1-mRFP together with RLP44-mTRQ2 and BRI1-mVEN led to a significant further decrease in the FLT of RLP44-mTRQ2 to $3.40 \mathrm{~ns}$, beyond the aforementioned values for the RLP44-mTRQ2/BRI1-mVEN heteromers of 3.54 ns (Fig. 5a). This is only explainable by the existence of an additional FRET pathway that becomes possible if RLP44, BAK1 and BRI1 have formed a ternary complex. When $10 \mathrm{nM}$ brassinolide (BL), an active brassinosteroid, was applied to the cells, no alteration in the FLT of RLP44mTRQ2 was observed (Fig. 5c). It was previously shown that FRET-FLIM is, in principle, independent of the donor concentration ${ }^{34}$. However, the relative amount of acceptor in comparison to the donor fluorophores (the donor-to-acceptor ratio) may influence the FLT of the donor ${ }^{19,21}$. Therefore, we investigated, whether this effect influences the FLT values obtained using different fluorescent protein ratios. As only the ratio of RLP44-mTRQ2/FLS2-mRFP and RLP44-mTRQ2/BAK1-mRFP exhibited a measurable difference within the RLP44-mTRQ2/BRI1-mVEN/FLS2-mRFP and the RLP44-mTRQ2/BRI1-mVEN/BAK1-mRFP in the three-fluorophore arrangements, differences in expression strength of the fusion proteins were not a major factor influencing the FLT of RLP44-mTRQ2 (SI Fig. 5).

In summary, we were able to confirm the ternary complex formation of RLP44, BAK1 and BRI1 by three-fluorophore FRET-FLIM in living plant cells. Furthermore, the FRET-FLIM approach is clearly superior to the intensity-based FRET approach, because the former is easier to implement and less prone to errors. 

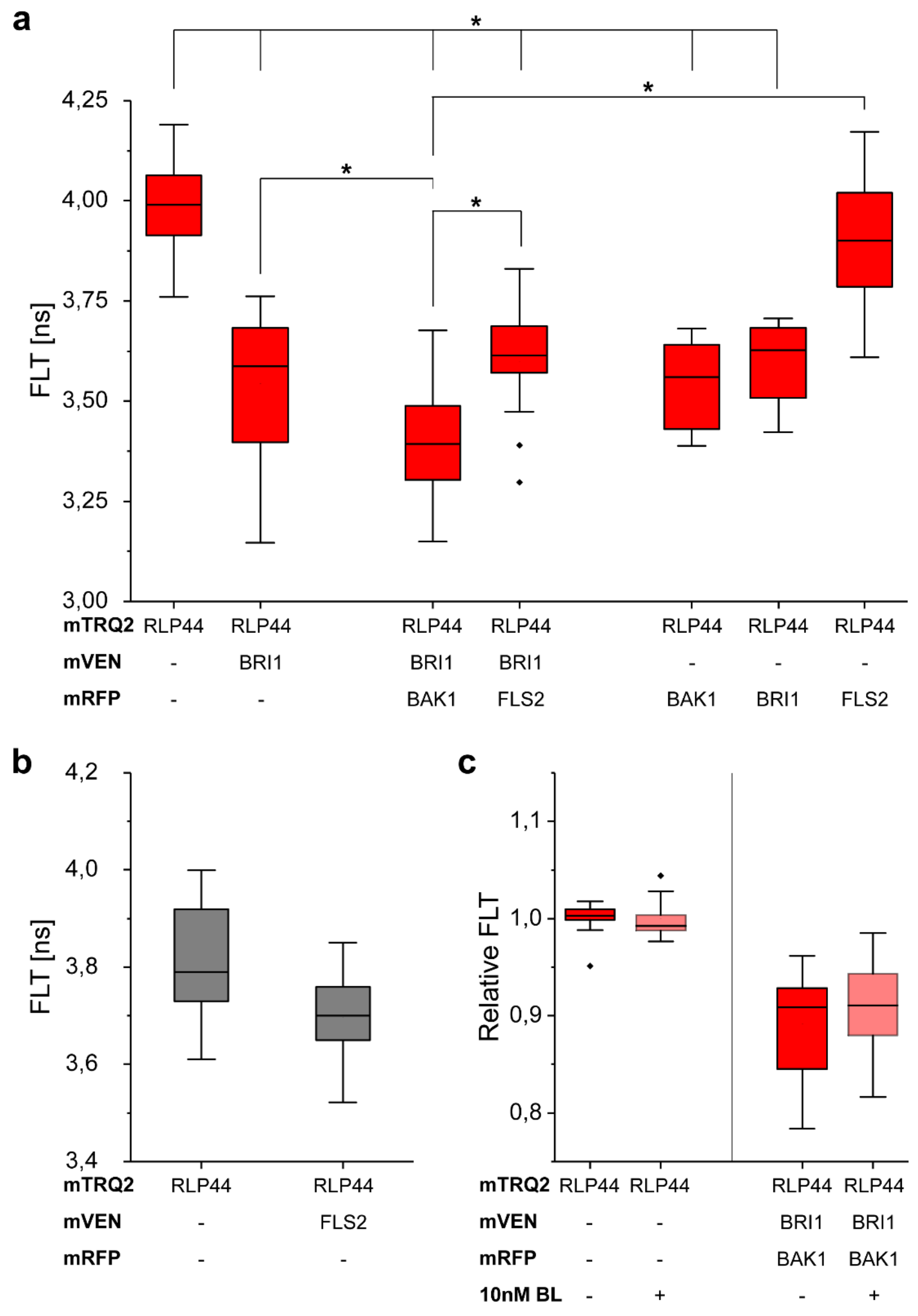
Fig. 5 | FRET-FLIM enables quantitative three-fluorophore protein-protein interaction and proximity analyses in $\boldsymbol{N}$. benthamiana leaf cells. a, Fluorescence lifetime (FLT) of RLP44-mTRQ2 after pulsed excitation of the cells with $440 \mathrm{~nm}$ light in the presence of the indicated $m V E N$ and $m R F P$ fusion proteins. $\mathbf{b}$, FLT of RLP44-mTRQ2 alone or in the presence of FLS2-mVEN. Data presentation as in a. c, Relative FLT of RLP44-mTRQ2 alone or in the presence of the indicated fusion proteins without (dark red) and with application of $10 \mathrm{nM}$ brassinolide (BL) (light red). The average FLT of RLP44-mTRQ2 in the absence of BL was set to 1.

The boxplots represent all data with the median as a solid black line within the box that is restricted by the first quartile (25\%; lower end) and the third quartile (75\%; upper end). Whiskers show the minimum and maximum value of the data, respectively, that are not defined as outlier (1.5 times interquartile range). Outliers are indicated as black diamonds. Statistical evaluations were performed by ANOVA followed by Tukey-Kramer HSD post hoc test. The black asterisk indicate statistically significant differences ( $p \leq=0.005)$. 


\section{Discussion}

Many studies in the non-plant field have independently established fluorophore sets for studying binary protein-protein interactions and complex formation in the cellular context ${ }^{35}$. Here we present three-fluorophore intensity-based FRET and FRET-FLIM approaches with the genetically encoded fluorophores mTRQ2, mVEN and mRFP in plant cells. The use of a blue, yellow and red fluorophores was the preferred application for three-fluorophore FRET studies, as it provides a good compromise between large Förster distances due to high spectral overlap and sufficient spectral separation for independent detection. A comparative study on available fluorophores in non-plant cells revealed that mTRQ2, YPet and mCherry is the most promising combination for three-fluorophore FRET ${ }^{35}$. However, YPet is not yet adapted for use in plant cells. In addition, even though mVEN is less bright than for instance YPet, it is a monomer ${ }^{36}$. We reasoned that the slightly lower brightness of mVEN is of less concern than the potential tendency of other fluorophores to form aggregates at high local concentrations in confined domains, which can be expected when they are fused to PM-resident proteins ${ }^{36,37}$. High brightness fluorophores, such as Ruby2 or TagRFPs, are outperformed by mCherry, that compensates its low brightness with its fast maturation rate ${ }^{38}$. The maturation time of mRFP, however, is also relatively short, which makes it an appropriate acceptor fluorophore as well. Until now, no study was able to circumvent direct FRET between the donor and the $2^{\text {nd }}$ acceptor for genetically encoded fluorophores ${ }^{21,38-42}$. Therefore, extensive corrections for the crosstalk of the fluorophores are required in intensity-based FRET. While organic dyes might have provided better photophysical properties, their application in plant cells is challenging as the cell wall interferes with in vivo labelling. Even in animal cells, the different labelling efficiencies of organic dyes are challenging for three-fluorophore FRET, as the correction for directly excited acceptor fluorescence proves to be problematic $34,43,44$.

As of yet, only one single study has tried to estimate which distances between three fluorophores represent the dynamic range of their set ${ }^{22}$. We think that a realistic estimation of the variability and restrictions of a three-fluorophore system is important for the interpretation of FRET data. For example, the general rule of thumb for reliable FRET measurements (below $10 \mathrm{~nm}$ ) may be imprecise or incorrect. Here, we calculated adapted distance limits, taking several factors into account: First, the spatial 
arrangement of the fluorophores in a three-fluorophore setting and secondly, the possibility for multiple acceptors per donor for higher complex stoichiometries. Indeed, for an ideal linear arrangement of the fluorophores mTRQ2, mVEN and mRFP, FRET measurements can cover distances of up to $13.1 \mathrm{~nm}$. This implies that FRET in membranes could not only occur between proteins within one and the same complex or domain, but might also be possible between proteins located in spatially distinct but adjacent domains (Fig. 6).
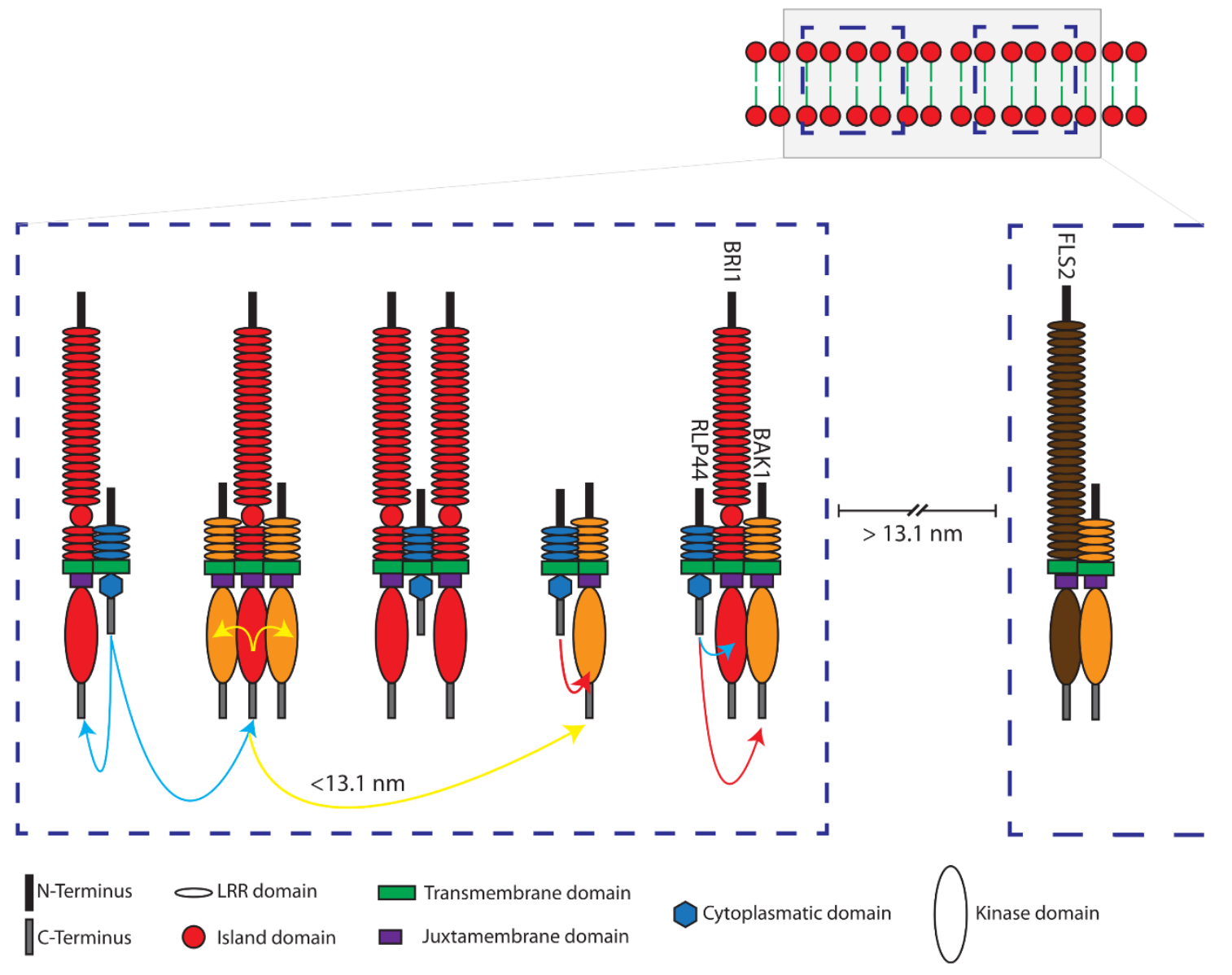

Fig. 6 | Model of the ternary interactions and spatial distances of RLP44, BRI1, BAK1 and FLS2 in defined nanodomains located in the plasma membrane of plant cells, as determined by three-fluorophore, intensity-based spectral FRET and FRET-FLIM. Shown are two distinct schematic nanodomains (blue dashed squares in the top corner) in the plasma membrane. The left nanodomain in the enlargement consists of RLP44-mTRQ2 (blue), BRI1-mVEN (red) and BAK1-RFP (orange) - for simplicity without the fluorophores and not in scale. The formation of different complexes is demonstrated by FRET between mTRQ2 and mVEN by blue, mVEN and mRFP by yellow and mTRQ2 and mRFP by red arrows. FRET is also possible between the fusion proteins of differently composed complexes, if they are in 
a distance of $\leq 13.1 \mathrm{~nm}$ (representative yellow arrow). Due to the absence of FRET, for instance, the BAK1-mVEN/FLS2-mRFP (brown) complex must be at least $13.1 \mathrm{~nm}$ apart from the nanodomain, that contains the RLP44 related complexes, and is very likely part of an independent nanodomain (right in the enlargement).

When assuming that the donor is located between the $1^{\text {st }}$ and $2^{\text {nd }}$ acceptor in a ternary complex, the likelihood of energy transfer from the donor via acceptor 1 to the acceptor 2 decreases, as the distance between acceptors 1 and 2 increases. These spatial arrangements should be considered when applying intensity-based FRET measurements. Furthermore, it is also recommended in this context to exchange at least one of the fluorophores between the fusion proteins, as it was done here. For classical donor-based FRET-FLIM, the differences in spatial organization may not be detectable, as no information is available on where the energy is transferred to. In principle, such spatial information can also be obtained using a more sophisticated FRET-FLIM approach, when mVEN is used as donor by excitation with another laser. In any case, FLIM-based FRET recordings have the advantage of a higher sensitivity, as the signal-to-noise ratio in intensity-based FRET measurements becomes worse after unmixing and sensitized emission calculations.

Since the plasma membrane is a compartment confined to two dimensions, the protein density is comparatively higher than in the three-dimensional case. In particular, membrane receptors tend to form complexes with higher stoichiometries, so that a donor can be surrounded by several acceptors, which can increase the apparent FRET efficiency. This can mask the principally linear relationship between EFRET and the affinity properties of the interacting fusion proteins. Therefore, no conclusions can be drawn about their affinity within a ternary complex and only limited information can be obtained about the absolute distances between the three fluorophores.

It was previously unclear whether RLP44 interacts with both BRI1 and BAK1 simultaneously. In this study, we applied intensity-based FRET and FRET-FLIM to prove that RLP44 is specifically located in close proximity (below $13.1 \mathrm{~nm}$ ) to both BRI1 and BAK1 in vivo. In the radius of $13.1 \mathrm{~nm}$, the three proteins can form trimers and/or be arranged as intermediate complexes (Fig. 6). Application of $10 \mathrm{nM}$ BL to the cells did not change the FRET characteristics in the spatial RLP44-mTRQ2/BRI1mVEN/BAK1-mRFP arrangement in the PM. This indicates that the RLP44/BAK1/BRI1 nanodomain is pre-formed in the PM in the absence of BL and 
none of the proteins appears to leave the nanodomain upon binding of BL to BRI1 to a significant amount.

According to our intensity-based FRET and FRET-FLIM recordings, FLS2 interacts with neither BRI1 nor RLP44, which is in agreement with recent BiFC studies ${ }^{30}$. Remarkably, the spatial distance between FLS2 and the RLP44-BAK1, RLP44-BRI1 and the RLP44-BRI1-BAK1 heteromers must be larger than $13.1 \mathrm{~nm}$. This indicates that FLS2 is localized in nanodomains that are spatially distinct from the RLP44consisting ones. Taking into account the nanometer accuracy of FRET, our approach decisively substantiates previous results, achieved by variable angle epifluorescence microscopy (VAEM) which has a physical resolution limit of roughly 250 to $300 \mathrm{~nm}^{31,33}$.

In summary, using the example of three selected chromophores, we show that threefluorophore intensity-based FRET and FRET-FLIM are techniques for analyzing the interaction and relative proximity of different proteins in the PM of living plant cells. Furthermore, by applying the presented calculations and experimental approaches, it is possible to distinguish between spatially distinct complexes and/or nanodomains in the PM that are different in their protein composition.

Although the two approaches are complementary, we strongly recommend the FRETFLIM approach rather than intensity-based, spectral FRET for ternary protein interaction and proximity analyses, as the latter is particularly vulnerable to changes in the donor-to-acceptor ratio. 


\section{Methods}

\section{Plasmid construction}

The cDNA sequence of the gene of interest without stop codon was brought into pDONR221-P3P2 (donor) or pDONR221-P1P4 (first acceptor) or pENTR TM/DTOPO ${ }^{\circledR}$ (second acceptor) as described by guidelines in the Gateway manual (Life Technologies) with primers listed in SI Table 3. The coding sequence of BRI1 and BAK1 was brought in the pENTR-D-TOPO previously ${ }^{7,8}$. For the generation of BRI1HA, primer previously published were used ${ }^{9}$ to bring CDS of BRI1 in pDONR207 and an LR with pGWB14 was performed. The LR into pB7RWG2 (RFP) ${ }^{45}$ and the 2in1 FRET vector pFRETtv-2in1-CC ${ }^{15}$ was performed as described previously ${ }^{15,46}$.

\section{Localization and FRET-FLIM studies}

Transformation of $N$. benthamiana was performed as described by ${ }^{15,47}$, omitting the washing step with sterile water. For transformations with multiple constructs, an $\mathrm{OD}_{600}$ of 0.1 was set and mixed 1:1:1 with silencing inhibitor p19. Plants were watered and left at ambient conditions $\left(24^{\circ} \mathrm{C}\right)$ with lid on top and imaged two days past transformation with an SP8 confocal laser scanning microscope (CLSM) (Leica Microsystems $\mathrm{GmbH}$, Wetzlar, Germany) with LAS AF and SymPhoTime software (Picoquant $\mathrm{GmbH}$, Berlin, Germany) using a 63x/1.20 water immersion objective 47,48. Data were derived from measurements of the lower epidermis, avoiding guard cells and stomata, with at least two biological replicates, comprising in average 20 data points and 11 data points for mTRQ2 - mRFP controls. Localization and quantification were performed with a minimum 3-fold line average for mTRQ2, mVEN and mRFP with the Argon laser set to $2 \%$ and excitations of $458 \mathrm{~nm} 40 \%, 514 \mathrm{~nm} 20 \%$ and $594 \mathrm{~nm}$ or $561 \mathrm{~nm} 30 \%$ and emission detection with 465-505 nm $400 \%$ on HyD, 525$565 \mathrm{~nm} 400 \%$ on SMD HyD and 605-650 nm $300 \%$ on SMD HyD, respectively.

FLIM measurements were performed with a $440 \mathrm{~nm}$ pulsed laser (LDH-P-C-470, Picoquant $\mathrm{GmbH}$, Berlin, Germany) with $40 \mathrm{MHz}$ repetition rate at a reduced scanning speed, yielding, with an image resolution of $256 \times 256$, a pixel dwell time of $\sim 20 \mu \mathrm{s}$. The maximal count rate was set to $\sim 2,000 \mathrm{cps}$. Measurements were stopped when the brightest pixel had a photon count of 500 . Only measurements with an even intensity distribution at the PM were included. The corresponding emission was detected with HyD SMD from $455 \mathrm{~nm}$ to $505 \mathrm{~nm}$ by time-correlated single-photon counting using a 
PicoHarp 300 module or a TimeHarp 260 module (PicoQuant GmbH, Berlin, Germany). The calculation of FLTs was performed by iterative reconvolution, i.e., the instrument response function was convolved with an exponential test functions to minimize the error with regard to the original TCSPC histograms in an iterative process. While the donor-only samples were fitted with mono-exponential decay functions, the energy transfer to fluorophores in the other samples resulted in additional decay rates. These histograms necessitated biexponential fitting functions, from which the fluorescent lifetime was derived by intensity weighted averaging. For the fastFLIM measurements, the maximal count rate was increased.

\section{Acquisition of $\lambda$-stacks (spectra)}

Expression of relevant fluorophores were checked via fluorescence level prior $\lambda$-stack acquisition. For $\lambda$-stacks, both sequential excitation and simultaneous excitation was used as mentioned in Results and an average of at least 6 ROIs of the PM with different expression levels of at least two biological replicates. At the Leica SP8 microscope, excitation at $458 \mathrm{~nm} 80 \%$ was used with SMD HyD 250 \%, measuring 460-625 nm with $\Delta 7.5 \mathrm{~nm}$ and $256 \times 256 \mathrm{px}$ resolution, a pixel dwell time of $\sim 20 \mu \mathrm{s}$ and three-fold line accumulation. At the Zeiss LSM880 (Carl Zeiss AG, Oberkochen, Germany) excitation at $485 \mathrm{~nm}$ with $30 \%$, NF458, $800 \mathrm{~V}$ of airy-scan detectors were used, measuring $460-650 \mathrm{~nm}$ or $560-650 \mathrm{~nm}$ with $\Delta 4.5 \mathrm{~nm}$ and 256x256 px resolution, speed 2 , digital gain set to 2 , pinhole set to 14.25 airy units and a threefold line accumulation. If over-all expression was very high, then for all samples measured that day line average was taken instead.

\section{Protein structures and sizes}

The intracellular domain of BRI1 (5LPW) and BAK1 (3TL8) as well as the fluorophore barrels were exported from the Protein Data Base (PDB). As viewer, Jmol: an opensource Java viewer for chemical structures in 3D ${ }^{49}$ with solvent accessible depiction was chosen and protein colors were changed. The secondary structure of linkers and the intracellular RLP44 domain was predicted with PEP-FOLD $3.5^{28}$, de-novo prediction, with standard settings and always model no1 (of 10) was used. Amino acid sequences were HPTFLYKVGQLLGTS for the donor-linker, NPAFLYKVVSRLGTS for the acceptor-linker, KGGRADPAFLYKVVIS for the second acceptor linker and 
CLWLRITEKKIVEEEGKISQSMPDY for RLP44cyto. The size of intracellular domains was calculated from the known distance of the alpha-barrel secondary structure, which is $\Delta 5.4 \AA$ from turn-to-turn and $4 \AA$ inner diameter.

\section{Statistics}

With one exception, each measurement was performed in at least three biological replicates. Each biological replica in turn included at least 3 individual recordings. In the exception, 2 biological replicates were performed, each containing 3 individual recordings. Images and plots were generated with Microsoft Excel v1809, SAS JMP 14 or MATLAB ${ }^{50}$, also using these programs for calculation of average, standard error (SE) and standard deviation (SD). To test for homogeneity of variance, Levene's test $(p<0.05)$ was employed and statistical significance for non-parametric distributions was calculated by a two-tailed, all-pair Kruskal-Wallis test followed by a Steel-Dwass post hoc correction using SAS JMP version 14.0.0 ${ }^{51}$. For small sample numbers in Fig. $3 d$ the 2-sample t-test was chosen ${ }^{52}$.

\section{Data availability}

All other data supporting the findings of the study are present in the main text and/or the Supplementary Information. Additional data related to this study are available from the corresponding authors upon request. Source data are provided with this paper.

\section{Code availability}

All MATLAB code for the calculation of the values in Table 1 and the spectral unmixing is available at https://github.com/svenzok/3F-FRET. 


\section{Acknowledgements}

The authors would like to thank F. Fässler for critical reading and F. de Courcy for English proofreading the manuscript. Research in our laboratories was supported by the German Research Foundation (DFG) with grants to S.Z.O.-K. (CRC 1101-Z02), to S.W. (WO 1660/6-1, WO 1660/6-2), to K.H. (HA 2146/22-1; HA 2146/22-2), to A.J.M. and Fra.W. (ME 1600/13-3) and to K.H. and A.J.M (CRC 1101-D02).

\section{Author contributions}

N.G., A.J.M. and K.H. conceived the project. N.G., S.Z.O.-K., Fri.W. and K.H. designed the study. N.G., S.z.O.-K., M.B., Fri.W. and EH performed the experiments. N.G., S.Z.O.-K. and L.R. conducted the mathematical and statistical analyses. N.G., S.z.O.K., L.R., Fra.W and Fri.W. analyzed the data. Fri.W. helped with the confocal experiments. Fra.W., A.J.M., K.H. and S.W. provided experimental materials and intellectual input. N.G., S.z.O.-K., L.R. and K.H. wrote the paper.

\section{References}

1. Gou, X. \& Li, J. Paired Receptor and Coreceptor Kinases Perceive Extracellular Signals to Control Plant Development. Plant Physiology 182, 1667-1681; 10.1104/pp.19.01343 (2020).

2. Wolf, S. Deviating from the Beaten Track: New Twists in Brassinosteroid Receptor Function. International journal of molecular sciences 21; 10.3390/ijms21051561 (2020).

3. Yin, Y. et al. A new class of transcription factors mediates brassinosteroidregulated gene expression in Arabidopsis. Cell 120, 249-259; 10.1016/j.cell.2004.11.044 (2005).

4. Vert, G. \& Chory, J. Downstream nuclear events in brassinosteroid signalling. Nature 441, 96-100; 10.1038/nature04681 (2006).

5. Mora-García, S. et al. Nuclear protein phosphatases with Kelch-repeat domains modulate the response to brassinosteroids in Arabidopsis. Genes \& development 18, 448-460; 10.1101/gad.1174204 (2004). 
6. Zhu, J.-Y. et al. The F-box Protein KIB1 Mediates Brassinosteroid-Induced Inactivation and Degradation of GSK3-like Kinases in Arabidopsis. Molecular cell 66, 648-657.e4; 10.1016/j.molcel.2017.05.012 (2017).

7. Caesar, K. et al. A fast brassinolide-regulated response pathway in the plasma membrane of Arabidopsis thaliana. The Plant Journal 66, 528-540;

10.1111/j.1365-313X.2011.04510.x (2011).

8. Witthöft, J. et al. The activation of the Arabidopsis P-ATPase 1 by the brassinosteroid receptor BRI1 is independent of threonine 948 phosphorylation. Plant Signaling \& Behavior 6, 1063-1066; 10.4161/psb.6.7.15650 (2014).

9. Wolf, S. et al. A receptor-like protein mediates the response to pectin modification by activating brassinosteroid signaling. Proceedings of the National Academy of Sciences of the United States of America 111, 15261-15266;

10.1073/pnas.1322979111 (2014).

10. Holzwart, E. et al. BRI1 controls vascular cell fate in the Arabidopsis root through RLP44 and phytosulfokine signaling. Proceedings of the National Academy of Sciences of the United States of America 115, 11838-11843;

10.1073/pnas.1814434115 (2018).

11.Förster, T. Zwischenmolekulare Energiewanderung und Fluoreszenz. Ann. Phys. 437, 55-75; 10.1002/andp.19484370105 (1948).

12. Noomnarm, U. \& Clegg, R. M. Fluorescence lifetimes. Fundamentals and interpretations. Photosynthesis research 101, 181-194; 10.1007/s11120-0099457-8 (2009).

13. Goedhart, J. et al. Structure-guided evolution of cyan fluorescent proteins towards a quantum yield of 93\%. Nature Communications 3, 751; 10.1038/ncomms1738 (2012).

14.Müller, S. M., Galliardt, H., Schneider, J., Barisas, B. G. \& Seidel, T. Quantification of Förster resonance energy transfer by monitoring sensitized emission in living plant cells. Frontiers in plant science 4, 413; 10.3389/fpls.2013.00413 (2013). 
15. Hecker, A. et al. Binary 2in1 Vectors Improve in Planta (Co)localization and Dynamic Protein Interaction Studies. Plant Physiology 168, 776-787; 10.1104/pp.15.00533 (2015).

16. Martin, K. J. et al. Accepting from the best donor; analysis of long-lifetime donor fluorescent protein pairings to optimise dynamic FLIM-based FRET experiments. PloS one 13, e0183585; 10.1371/journal.pone.0183585 (2018).

17. Miyawaki, A. \& Tsien, R. Y. in Cell biology and physiology, edited by J. Thorner (Acad. Press, San Diego, Calif., 2000), pp. 472-500.

18. Nagai, T. et al. A variant of yellow fluorescent protein with fast and efficient maturation for cell-biological applications. Nature Biotechnology 20, 87-90; 10.1038/nbt0102-87 (2002).

19.Bunt, G. \& Wouters, F. S. FRET from single to multiplexed signaling events. Biophysical reviews 9, 119-129; 10.1007/s12551-017-0252-z (2017).

20.Wouters, F. S. Förster Resonance Energy Transfer and Fluorescence Lifetime Imaging (2017).

21.Sun, Y., Wallrabe, H., Booker, C. F., Day, R. N. \& Periasamy, A. Three-color spectral FRET microscopy localizes three interacting proteins in living cells. Biophysical journal 99, 1274-1283; 10.1016/j.bpj.2010.06.004 (2010).

22. Haustein, E., Jahnz, M. \& Schwille, P. Triple FRET. A tool for studying long-range molecular interactions. Chemphyschem : a European journal of chemical physics and physical chemistry 4, 745-748; 10.1002/cphc.200200634 (2003).

23. Koushik, S. V., Blank, P. S. \& Vogel, S. S. Anomalous Surplus Energy Transfer Observed with Multiple FRET Acceptors. PloS one 4, e8031; 10.1371/journal.pone.0008031 (2009).

24.Rekas, A., Alattia, J.-R., Nagai, T., Miyawaki, A. \& Ikura, M. Crystal structure of venus, a yellow fluorescent protein with improved maturation and reduced environmental sensitivity. J. Biol. Chem. 277, 50573-50578;

10.1074/jbc.M209524200 (2002).

25. Yan, L. et al. Structural basis for the impact of phosphorylation on the activation of plant receptor-like kinase BAK1. Cell Research 22, 1304-1308; 10.1038/cr.2012.74 (2012). 
26.Bojar, D. et al. Crystal structures of the phosphorylated BRI1 kinase domain and implications for brassinosteroid signal initiation. The Plant journal : for cell and molecular biology 78, 31-43; 10.1111/tpj.12445 (2014).

27.Großeholz, R. et al. Specifying the role of BAK1-interacting receptor-like kinase 3 in brassinosteroid signaling. Journal of integrative plant biology; 10.1111/jipb.12803 (2019).

28. Lamiable, A. et al. PEP-FOLD3: faster de novo structure prediction for linear peptides in solution and in complex. Nucleic acids research 44, W449-54; 10.1093/nar/gkw329 (2016).

29.Nam, K. H. \& Li, J. BRI1/BAK1, a Receptor Kinase Pair Mediating Brassinosteroid Signaling. Cell 110, 203-212; 10.1016/S0092-8674(02)00814-0 (2002).

30.Garnelo Gomez, B., Lozano-Duran, R. \& Wolf, S. Phosphorylation-dependent routing of RLP44 towards brassinosteroid or phytosulfokine signalling. Supplemental Material (2019).

31.Bücherl, C. A. et al. Plant immune and growth receptors share common signalling components but localise to distinct plasma membrane nanodomains. eLife 6; 10.7554/eLife.25114 (2017).

32.Galperin, E., Verkhusha, V. V. \& Sorkin, A. Three-chromophore FRET microscopy to analyze multiprotein interactions in living cells. Nature Methods $\mathbf{1}$, 209 EP -; 10.1038/nmeth720 (2004).

33. Hochreiter, B., Garcia, A. P. \& Schmid, J. A. Fluorescent proteins as genetically encoded FRET biosensors in life sciences. Sensors (Basel, Switzerland) 15, 26281-26314; 10.3390/s151026281 (2015).

34.Becker, W. Fluorescence lifetime imaging--techniques and applications. Journal of microscopy 247, 119-136; 10.1111/j.1365-2818.2012.03618.x (2012).

35.Scott, B. L. \& Hoppe, A. D. Optimizing fluorescent protein trios for 3-Way FRET imaging of protein interactions in living cells. Scientific Reports 5, 10270 EP -; 10.1038/srep10270 (2015).

36.Bajar, B. T., Wang, E. S., Zhang, S., Lin, M. Z. \& Chu, J. A Guide to Fluorescent Protein FRET Pairs. Sensors (Basel, Switzerland) 16; 10.3390/s16091488 (2016). 
37. Miyawaki, A. Development of probes for cellular functions using fluorescent proteins and fluorescence resonance energy transfer. Annual Review of Biochemistry 80, 357-373; 10.1146/annurev-biochem-072909-094736 (2011).

38. Hoppe, A. D., Scott, B. L., Welliver, T. P., Straight, S. W. \& Swanson, J. A. N-way FRET microscopy of multiple protein-protein interactions in live cells. PloS one $\mathbf{8}$, e64760; 10.1371/journal.pone.0064760 (2013).

39.He, L., Wu, X., Simone, J., Hewgill, D. \& Lipsky, P. E. Determination of tumor necrosis factor receptor-associated factor trimerization in living cells by CFP-YFPmRFP FRET detected by flow cytometry. Nucleic acids research 33, e61; 10.1093/nar/gni057 (2005).

40.Kuo, H.-L., Ho, P.-C., Huang, S.-S. \& Chang, N.-S. Chasing the signaling run by tri-molecular time-lapse FRET microscopy. Cell Death Discovery 4, 45; 10.1038/s41420-018-0047-4 (2018).

41.Pauker, M. H., Hassan, N., Noy, E., Reicher, B. \& Barda-Saad, M. Studying the dynamics of SLP-76, Nck, and Vav1 multimolecular complex formation in live human cells with triple-color FRET. Science signaling 5, rs3;

10.1126/scisignal.2002423 (2012).

42.Wallrabe, H. et al. IQGAP1 interactome analysis by in vitro reconstitution and live cell 3-color FRET microscopy. Cytoskeleton (Hoboken, N.J.) 70, 819-836; 10.1002/cm.21146 (2013).

43.Fábián, Á., Horváth, G., Vámosi, G., Vereb, G. \& Szöllősi, J. TripleFRET measurements in flow cytometry. Cytometry. Part $A$ : the journal of the International Society for Analytical Cytology 83, 375-385; 10.1002/cyto.a.22267 (2013).

44.Fazekas, Z. et al. Two-sided fluorescence resonance energy transfer for assessing molecular interactions of up to three distinct species in confocal microscopy. Cytometry. Part A : the journal of the International Society for Analytical Cytology 73, 209-219; 10.1002/cyto.a.20489 (2008).

45. Karimi, M., Inzé, D. \& Depicker, A. GATEWAY vectors for Agrobacteriummediated plant transformation. Trends in plant science 7, 193-195 (2002). 
46. Grefen, C. \& Blatt, M. R. A 2in1 cloning system enables ratiometric bimolecular fluorescence complementation (rBiFC). BioTechniques 53, 311-314; 10.2144/000113941 (2012).

47. Ladwig, F. et al. Phytosulfokine Regulates Growth in Arabidopsis through a Response Module at the Plasma Membrane That Includes CYCLIC NUCLEOTIDE-GATED CHANNEL17, H+-ATPase, and BAK1. The Plant cell 27, 1718-1729; 10.1105/tpc.15.00306 (2015).

48. Mohrholz, A. et al. The striking flower-in-flower phenotype of Arabidopsis thaliana Nossen (No-0) is caused by a novel LEAFY allele. Supplemental Figures and Tables (2019).

49.Jmol: an open-source Java viewer for chemical structures in 3D. Available at http://www.jmol.org/.

50.MATLAB. 9.8.0.1417392 (R2020a) (The MathWorks Inc, Natick, Massachusetts, 2020).

51.Ohmi, Y. et al. Sialylation converts arthritogenic IgG into inhibitors of collageninduced arthritis. Nature Communications 7, 11205; 10.1038/ncomms11205 (2016).

52. de Winter, J. C .F. Using the Student's "t"-Test with Extremely Small Sample Sizes. Practical Assessment, Research \& Evaluation 18 (2013). 


\section{Three-fluorophore FRET enables the analysis of ternary protein association in living plant cells}

Nina Glöckner ${ }^{\mathrm{a}}$, Sven zur Oven-Krockhaus ${ }^{\mathrm{a}, \mathrm{b}}$, Leander Rohr ${ }^{\mathrm{a}}$, Frank Wackenhut ${ }^{\mathrm{b}}$, Moritz Burmeister ${ }^{\mathrm{b}}$, Friederike Wanke ${ }^{\mathrm{a}}$, Eleonore Holzwart ${ }^{\mathrm{c}}$, Alfred J. Meixner ${ }^{\mathrm{b}}$, Sebastian Wolf ${ }^{\mathrm{c}}$ and Klaus Harter ${ }^{\mathrm{a}^{*}}$

${ }^{a}$ Center for Plant Molecular Biology, University of Tübingen, Tübingen, Germany

'Institute for Physical \& Theoretical Chemistry, University of Tübingen, Tübingen, Germany

${ }^{c}$ Centre for Organismal Studies, University of Heidelberg, Heidelberg, Germany

*corresponding author (Email: klaus.harter@zmbp.uni-tuebingen.de)

\section{Supplementary Information}

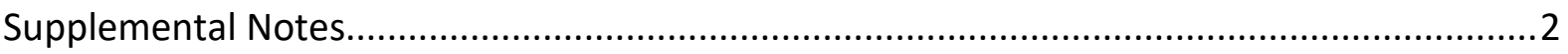

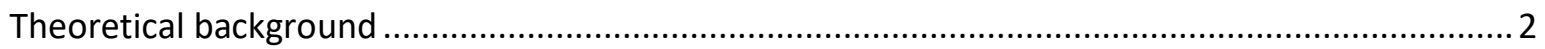

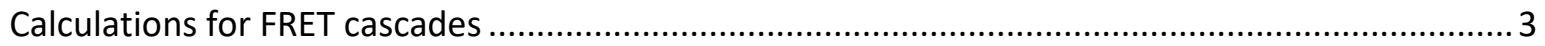

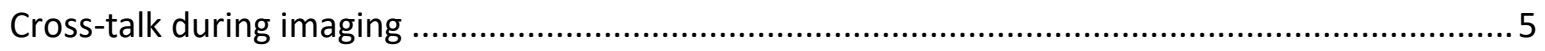

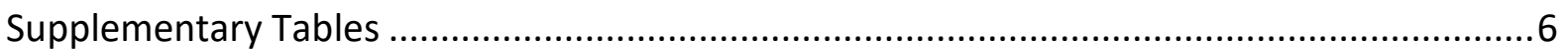

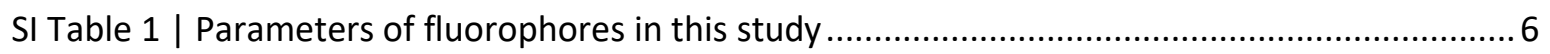

SI Table 2 | Coefficients for spectral bleed-through (bt) and cross-excitation (ce) ........................... 6

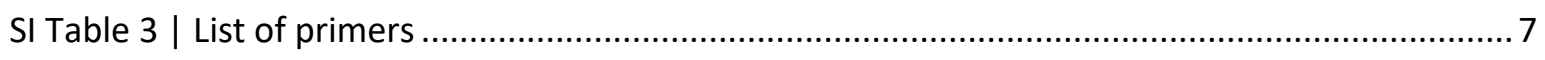

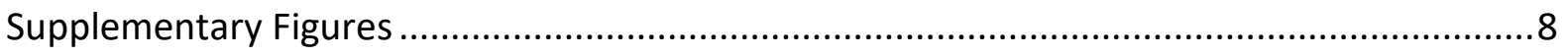

SI Figure 1 | RLP44-mTRQ2, BRI1-mVEN and BAK1-mRFP are expressed together N. benthamiana epidermal leaf cells.

SI Figure 2 | Wavelength-dependent intensity measurements reveal different fusion protein expression levels for different plasma membrane regions in transiently transformed $\mathrm{N}$. benthamiana epidermal leaf cells.

SI Figure 3| BRI ${ }^{\mathrm{HA}}$ is expressed in transiently transformed $\mathrm{N}$. benthamiana leaf cells...................11

SI Figure 4 | FLS2 does not interfere with the RLP44-related interactions (extension to Fig. 4).....12

SI Figure 5 | The overall donor to acceptor ratios is not significantly different in the protein fusion

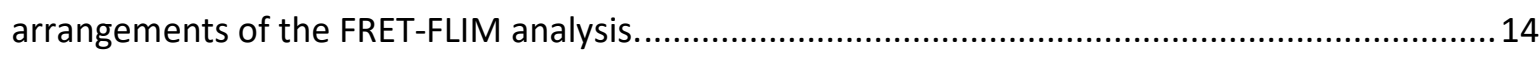

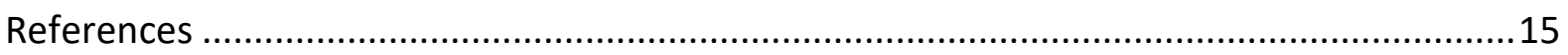




\section{Supplemental Notes}

\section{Theoretical background}

The FRET efficiency $(E)$ between a donor $(D)$ and an acceptor (A) fluorophore is given by

$$
E=\frac{R_{0}^{6}}{R_{0}^{6}+r^{6}}
$$

with $r$ as the distance between donor and acceptor and $R_{0}$ as the distance for $50 \%$ energy transfer (Förster distance):

$$
R_{0}(n m)=0.02108 \cdot\left(\kappa^{2} \cdot n^{-4} \cdot Q Y_{D} \cdot J\right)^{1 / 6}
$$

with $\kappa^{2}$ as the dipole orientation factor, $n$ as the refractive index of the medium, $Q Y_{D}$ as the quantum yield of the donor and $J$ as the spectral overlap between donor emission and acceptor absorption:

$$
J=\varepsilon_{A} \frac{\int f_{D}(\lambda) f_{A}(\lambda) \lambda^{4} d \lambda}{\int f_{D}(\lambda) d \lambda}
$$

with $\varepsilon_{A}$ as the molar attenuation coefficient of the acceptor at the peak absorption wavelength, $\lambda$ as the wavelength in $\mathrm{nm}, f_{D}$ and $f_{A}$ as the normalized donor emission and acceptor absorption spectra, respectively.

As evident in equation (2), the dipole orientation factor $\kappa^{2}$ has a strong influence on the calculated $R_{0}$ and thus $E$ :

$$
\kappa^{2}=\cos ^{2} \omega\left(1+3 \cos ^{2} \theta\right)
$$

Here, $\omega$ is the angle between the electric field vector of $D$ at the location of $A$ and the absorption dipole orientation of $A$ and $\theta$ is the angle between the emission dipole of $D$ and the separation vector of D-A (Lakowicz 2006).

For the standard assumption of $\kappa^{2}=2 / 3$ to be true, the rotational diffusion of a fluorophore has to be faster than the fluorescence lifetime (FLT) of D (Müller et al. 2013; Hink et al. 2002). However, this may not be true for genetically-encoded fluorescent proteins used in FRET studies for several reasons: (i) The fluorophore barrel is large and has a rotational correlation time of about 20-30 ns, whereas the FLT is in a range of 1-4 ns (Vogel et al., 2012). (ii) The fluorophores are attached to the proteins of interest with a flexible linker (Chen et al. 2013; van Rosmalen et al. 2017; George and Heringa 2002; Chen et al. 2013). In previous studies, a linker length of 15 amino acids was assumed to allow free rotation of the fluorophore, even though this may not be fully true (Ujlaky-Nagy et al. 2018; Szöllosi et al. 2006; Shrestha et al. 
201). But as no better options are available, the standard assumption is used. This introduces an error due to different fluorophore orientations. Hink et al. (2002) proposed to use $\kappa^{2}=0.476$, which is the value of the orientation factor for a rigid, randomized ensemble of D-A pairs (Steinberg 1971). This effectively reduces the calculated Förster distance. It does not eliminate the possibility of specifically existent deviations due to fixed protein arrangements, e.g., preventing FRET or other spatial arrangements. It is important to keep in mind that the FRET efficiency does not correspond to fixed real distances (Müller et al. 2013): The presence of FRET always means that they are in close proximity, but how close exactly can rarely be precisely determined, especially in live-cell imaging. The distance $r$ that is accessible through FRET-measurements is in average between $0.5 R_{0} \leq r \leq 1.5 R_{0}$ (Gadella 2009; Müller et al. 2013). Most FRET pairs have Förster distances between 4 and 7 ns (Bajar et al. 2016; Mastop et al. 2017). As a rule of thumb, FRET is restricted to distances below $10 \mathrm{~nm}$. The absence of FRET does not necessarily mean that the proteins of interest are not in close proximity or do not interact, e.g., due to unfavorable fluorophore attachment positions. Three-fluorophore FRET-FLIM, in turn, has the same limitations.

\section{Calculations for FRET cascades}

We define a cascading, linear three-chromophore FRET system

$$
1-2-3
$$

with 3 as the most redshifted fluorophore. When 2 acts as the donor, the spectral overlap of 2's emission spectrum with 1's absorption spectrum is negligible. The FRET efficiency for the path $2 \rightarrow 3$ can, therefore, be calculated with (1) as follows:

$$
E_{23}=\frac{R_{0_{23}}^{6}}{R_{0_{23}}^{6}+r^{6}{ }_{23}} \text {. }
$$

When 1 acts as the donor, two paths are possible:

$$
\begin{gathered}
1 \rightarrow 2 \rightarrow 3 \text { (energy transfer to } 3 \text { via } 2 \text { ) or } \\
1 \rightarrow 3 \text { (direct energy transfer to } 3 \text { ). }
\end{gathered}
$$

The energy can follow either path, so the energy transfer possibility for $1 \longrightarrow 2$ is diminished by the energy transfer possibility for $1 \longrightarrow 3$ and vice versa. This decrease can be expressed in terms of the fluorophore's quantum yield, using the following equations by Liu \& Lu (2002). For $1 \rightarrow 2:$ 


$$
\Delta \mathrm{QY}_{12}=\frac{R_{0_{13}}^{6}}{R_{0_{13}}^{6}+r^{6}{ }_{13}}
$$

$R_{0}$ to the power of 6 contains $Q Y$ as a multiplicator (cf. (2)), so the lost fraction of $Q Y$ can be implemented in $R_{0}$ as follows:

$$
R_{0_{12}}^{6}{ }^{\prime}=R_{0_{12}}^{6} \cdot\left(1-\Delta \mathrm{QY}_{12}\right)=R_{0_{12}}^{6} \cdot\left(1-\frac{R_{0_{13}}^{6}}{R_{0_{13}}^{6}+r^{6}{ }_{13}}\right),
$$

with $R_{0_{12}}^{6}{ }^{\prime}$ and $R_{0}^{6}{ }_{12}$ as the Förster distance of $1 \rightarrow 2$, when 3 is present or absent, respectively.

This adjusted Förster distance now replaces the numerator in (1):

$$
E_{12}=\frac{R_{0_{12}}^{6}}{R_{0_{12}}^{6}+r^{6}{ }^{\prime}}
$$

Combining and simplifying (6) and (7):

$$
E_{12}=\frac{\left(R_{0_{12}} \cdot r_{13}\right)^{6}}{\left(R_{0_{12}} \cdot r_{13}\right)^{6}+\left(R_{0_{13}} \cdot r_{12}\right)^{6}+\left(r_{12} \cdot r_{13}\right)^{6}}
$$

A similar result can be acquired for $1 \rightarrow 3$ :

$$
E_{13}=\frac{\left(R_{0_{13}} \cdot r_{12}\right)^{6}}{\left(R_{0_{13}} \cdot r_{12}\right)^{6}+\left(R_{0_{12}} \cdot r_{13}\right)^{6}+\left(r_{12} \cdot r_{13}\right)^{6}}
$$

The overall FRET efficiency for the above $1 \rightarrow 2 \rightarrow 3$ FRET cascade can now be estimated, using (5), (9) and (10):

$$
E_{\text {casc. }}=E_{12} \cdot E_{23}+E_{13}
$$

In case of a crowded system like a planar biological membrane, the possibility of one donor interacting with multiple acceptors is much higher, changing (1) to:

$$
E_{\text {mult.acc. }}=\frac{R_{0}^{6}}{R_{0}^{6}+\left(\frac{1}{n_{a}}\right) \cdot r^{6}}
$$

with $n_{a}$ as the number of acceptors per donor, also known as the antenna effect (Bunt et al., 2017). Moreover, the FRET surplus effect changes the average $\kappa^{2}$ value due to 
selection bias (Bunt et al., 2017; Koushik et al., 2009) that in turn influences the operational $R_{0}$ value. Using this adaptation for multiple acceptors, the overall FRET efficiency could now be calculated following the above equations leading to (11). However, this essentially mixes a two-dimensional approach with the very simplified assumption of a linear fluorophore arrangement. Moreover, even the linear FRET cascade approach neglects, e.g., the arrangement $2-1-3$. The truth will be much more complicated, including complex geometric arrangements of the fluorophores, enabling a multitude of possible energy transfer pathways that will not be solved easily without performing extensive numerical simulations. However, this set of equations and the derived results illustrate how a third fluorophore changes the FRET working parameters and how important it is to consider the actual biological situation - in this example, the analysis of (typically densely packed) protein complexes in the plasma membrane, which is of great common interest in the life science community. Although these theoretical considerations can only be simplified approximations, they impressively show that FRET interaction assays can, under certain conditions, cover much larger distances than generally expected, making this method viable for a much broader range of analyses.

\section{Cross-talk during imaging}

Cross-talk in the form of spectral bleed through (bt) and cross-excitation (ce) was present for our fluorophores. To account for this, the bt and ce was assessed from normalized absorption and emission spectra and quantified by imaging single-fluorophore expressing $N$. benthamiana plants.

Excitation at $458 \mathrm{~nm}$ lead to a relative absorption of $91 \%$ for $\mathrm{mTRQ} 2$ and a cross-excitation of $8 \%$ for mVEN and 3\% for mRFP (Fig. 1A purple, vertical line). Excitation at $514 \mathrm{~nm}$ for mVEN yielded a relative absorption of $99 \%$ and a cross-excitation of mRFP of $20 \%$ (Fig. 1A green line). mRFP was excited with $561 \mathrm{~nm}$ with a relative absorption of $61 \%$ (Fig. 1A orange line). Spectral bleed-through was present from mTRQ2 to mVEN and from mVEN to MRFP: When detecting mVEN with a bandwidth from 525 to $565 \mathrm{~nm}, 35 \%$ of the signal originates from mTRQ2 (SI Fig. 2, SI Table 2). Detection of mRFP between 605 and $650 \mathrm{~nm}$ included $6 \%$ of mVEN emission (SI Fig. 2, SI Table 2). When quantifying the FI, also the molecular brightness of a fluorophore, detection settings such as detector gain and laser strength are influencing the amount of bleed-through and cross-talk. Thus, we calculated the coefficients from 
imaging single-labelled samples with sequential excitation (SI Table 2). Measured bt and ce from $\mathrm{mTRQ} 2$ to the yellow and red channel was higher than calculated, as higher laser settings were used to excite mTRQ2. Measured ce from mVEN to the red channel was in the same order of magnitude as calculated. As the molecular brightness of mVEN is relatively high (SI Table 1), the bt to the red channel was higher than calculated (SI Table 2), even with reduced laser power for $514 \mathrm{~nm}$ compared to the $458 \mathrm{~nm}$ and $561 \mathrm{~nm}$ laser lines (see Material and methods).

\section{Supplementary Tables}

\section{SI Table 1 | Parameters of fluorophores in this study}

Quantum yield (QY), Molar attenuation coefficient $(\varepsilon)$ at the absorbance maximum, Brightness as the product QY. $\varepsilon$, Maturation half-time at $37^{\circ} \mathrm{C}$ in E. coli $\left(t_{50}\right)$, Photostability as half-time of bleaching under laser scanning illumination with $80 \mu \mathrm{W}\left(\mathrm{t}_{1 / 2}\right), \mathrm{pH}$ stability $\left(\mathrm{pK}_{\mathrm{a}}\right)$ and proportion of monomeric state. For comparison: EGFP features a brightness of $33.6 \mathrm{mM}^{-1} \mathrm{~cm}^{-1}$, a bleaching half-time of $159.7 \mathrm{~s}$ and a $\mathrm{pK}_{\mathrm{a}}$ of 6.0 .

\begin{tabular}{cccccccc}
\hline & QY & $\begin{array}{c}\varepsilon \\
{\left[\mathrm{mM}^{-1} \mathrm{~cm}^{-1}\right]}\end{array}$ & $\begin{array}{c}\text { Brightness } \\
{\left[\mathrm{mM}^{-1} \mathrm{~cm}^{-1}\right]}\end{array}$ & $\begin{array}{c}\text { Maturation } \\
\text { half-time } \mathbf{t}_{50} \\
{[\mathbf{m i n}]}\end{array}$ & $\begin{array}{c}\text { Photostability } \\
\mathbf{t} / 2[\mathrm{~s}]\end{array}$ & $\begin{array}{c}\mathrm{pH} \\
\text { stability } \\
\mathbf{p K}_{\mathbf{a}}\end{array}$ & $\begin{array}{c}\text { monomer } \\
{[\%]}\end{array}$ \\
\hline mTRQ2 & 0.93 & 30 & 27.9 & 33.5 & 71.7 & 3.1 & 93.8
\end{tabular}

(Goedhart et al. 2012; Cranfill et al. 2016; Balleza et al. 2018)

$\begin{array}{llllllll}\text { mVEN } & 0.64 & 105 & 67.2 & 17.6 & 26.5 & 5.5 & 83.9\end{array}$

(Kremers et al. 2006; Cranfill et al. 2016; Balleza et al. 2018)

$\begin{array}{llllllll}\text { mRFP } & 0.25 & 44 & 11.0 & 21.9 & 26.3 & 4.5 & 95.8\end{array}$

(Campbell et al. 2002; Cranfill et al. 2016; Balleza et al. 2018)

SI Table 2 | Coefficients for spectral bleed-through (bt) and cross-excitation (ce)

The bt and ce coefficients with standard error (SE) for relevant laser lines were both measured in images when only the relevant fluorophore was expressed (D:A1:A2) and additionally calculated based on normalized spectra.

\begin{tabular}{lccc|ccc}
\hline & \multicolumn{3}{c|}{ bleed-through (bt) } & \multicolumn{3}{c}{ cross-excitation (ce) } \\
& $1>>2$ & $1>>3$ & $2>>3$ & $1>>2$ & $1>>3$ & $2>>3$ \\
\hline Average false/true signal & 0.41 & 0.04 & 0.14 & 0.11 & 0.03 & 0.16 \\
SE & 0.01 & 0.001 & 0.02 & 0.01 & 0.01 & 0.01 \\
Ratio D:A1:A2 & $1: 0: 0$ & $1: 0: 0$ & $0: 1: 0$ & $0: 1: 0$ & $0: 0: 1$ & $0: 0: 1$ \\
Excitation laser [nm] & 458 & 458 & 514 & 458 & 458 & 514 \\
Calculated false/true signal & 0.35 & 0.01 & 0.06 & 0.08 & 0.03 & 0.2
\end{tabular}


SI Table 3 | List of primers

\begin{tabular}{|c|c|c|}
\hline GOI - vector & Forward primer $5^{\prime} \| 3^{\prime}$ & Reverse primer $5^{\prime} \| 3^{\prime}$ \\
\hline RLP44 - & GGGGACAACTTTGTATAATAAAGTTGtaA & GGGGACCACTTTGTACAAGAAAGCTGGG \\
\hline $\begin{array}{l}\text { pDONR221- } \\
\text { P3P2 }\end{array}$ & TGACAAGGAGTCACCGGTTAC & TtGTAATCAGGCATAGATTGAC \\
\hline BRI1 - & GGGGACAAGTTTGTACAAAAAAGCAGGC & GGGGACAACTTTGTATAGAAAAGTTGGG \\
\hline $\begin{array}{l}\text { pDONR221- } \\
\text { P1P4 }\end{array}$ & TtaATGAAGACTTTTTCAAGCTTCTT & TGTAATTTTCCTTCAGGAACTTCTT \\
\hline FLS2 - & GGGGACAAGTTTGTACAAAAAAGCAGGC & GGGGACAACTTTGTATAGAAAAGTTGGG \\
\hline $\begin{array}{l}\text { pDONR221- } \\
\text { P1P4 }\end{array}$ & TtaATGAAGTTACTCTCAAAGAC & TGAACTTCTCGATCCTCGTTACG \\
\hline FLS2 - & GGGGACAAGTTTGTACAAAAAAGCAGGC & GGGGACCACTTTGTACAAGAAAGCTGGG \\
\hline pDONR207 & TtaATGAAGTTACT & TgAACTTCTCGATCCT \\
\hline
\end{tabular}




\section{Supplementary Figures}

Detection channel

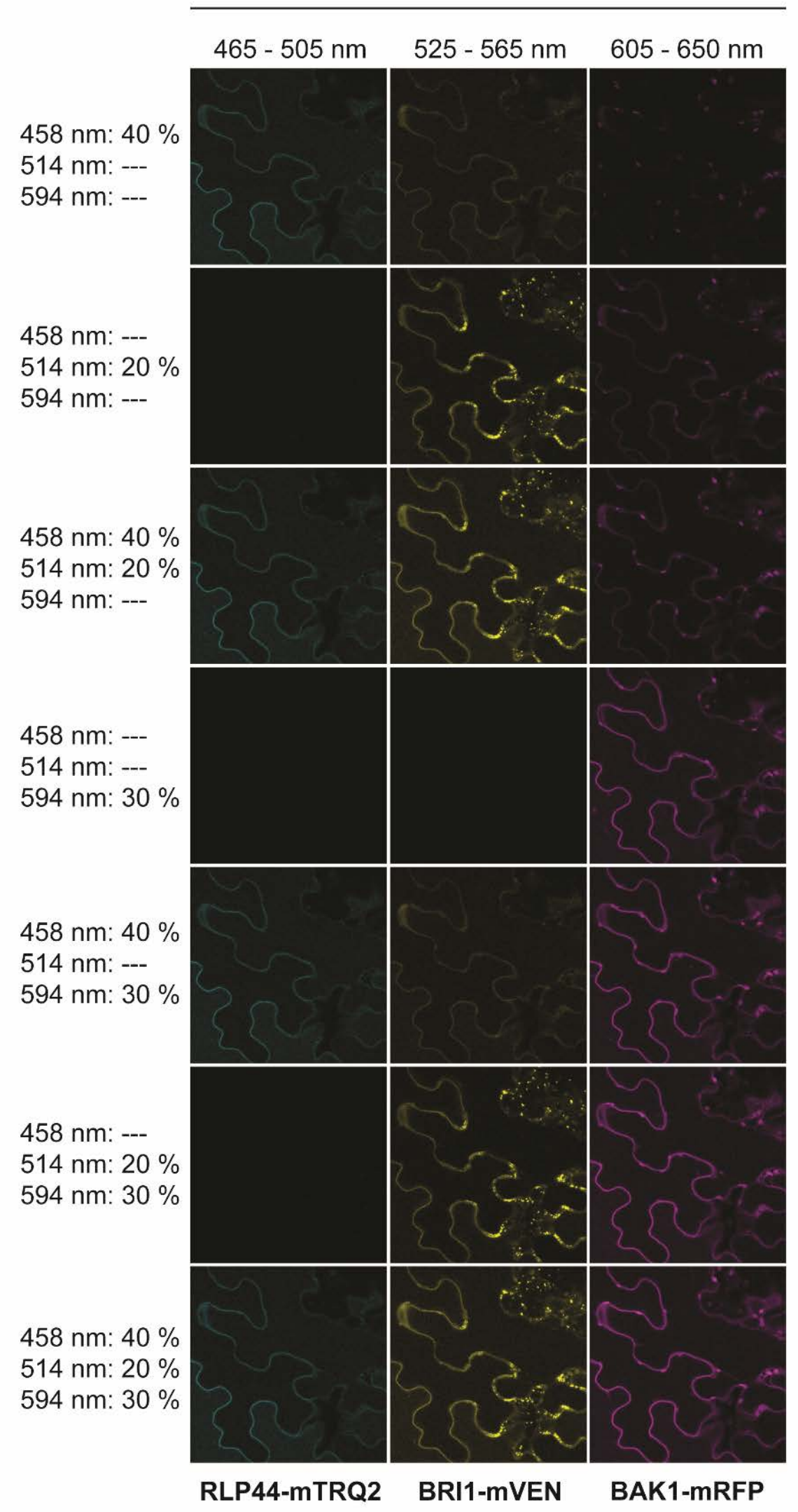


SI Figure 1 | RLP44-mTRQ2, BRI1-mVEN and BAK1-mRFP are expressed together in N. benthamiana epidermal leaf cells.

Representative confocal images of the fluorescence intensity in the MTRQ2 channel, the mVEN channel and the mRFP channel are exemplary shown for the combination of RLP44-mTRQ2, BRI1mVEN and BAK1-mRFP two days after transient transformation of $N$. benthamiana leaf cells. The excitation wavelengths and the relative laser intensities are depicted to the left. The blue channel for detection of RLP44-mTRQ was 465-505 nm, the yellow channel for detection of BRI1-mVEN was 525$565 \mathrm{~nm}$ and the red channel for detection of mRFP was 605-650 nm. The scale bar represents $20 \mu \mathrm{m}$. In general, before the acquisition of spectra or the fluorescence intensities, the expression of each fusion construct was determined by sequential excitation, meaning that one laser line was switched on at a time. 
a

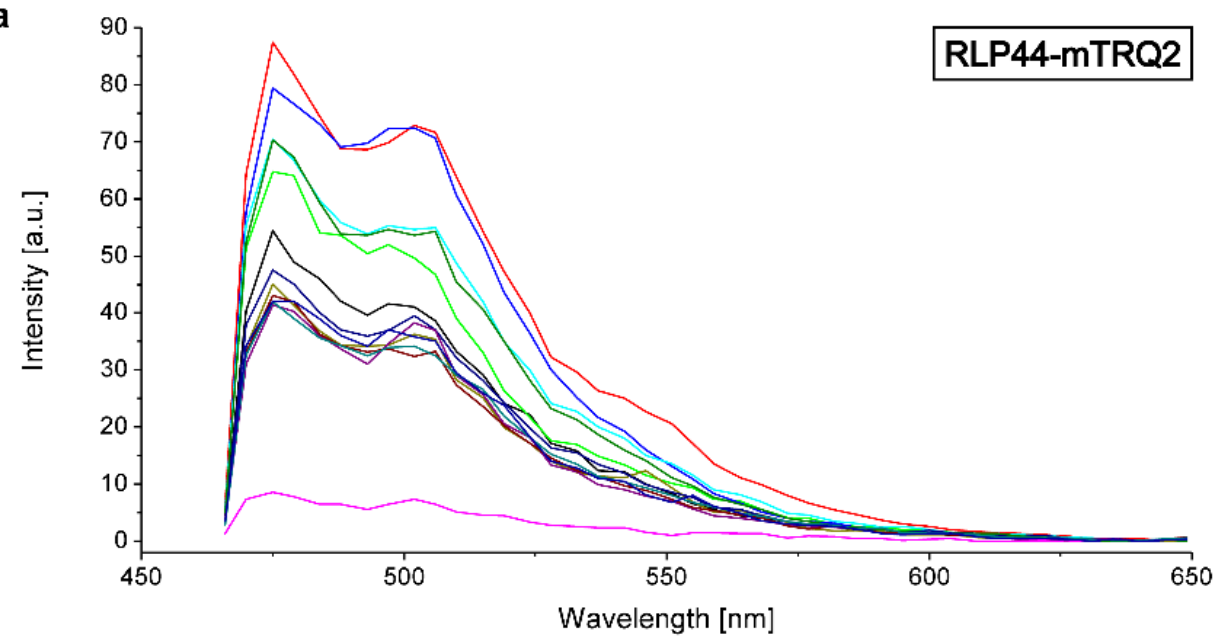

b

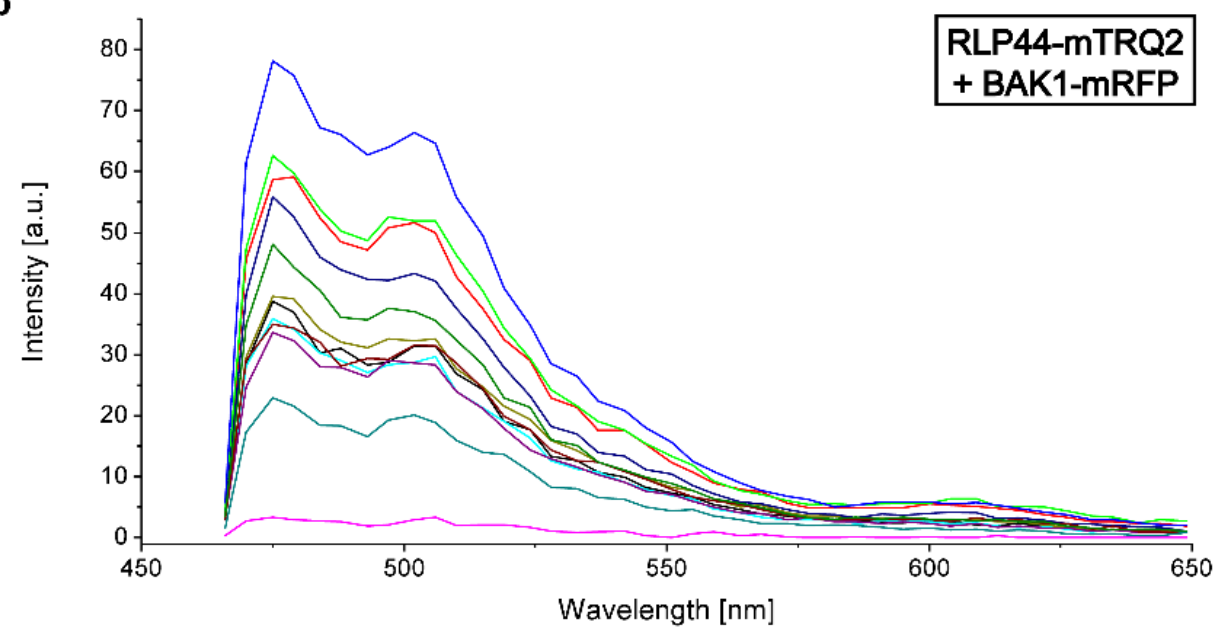

C

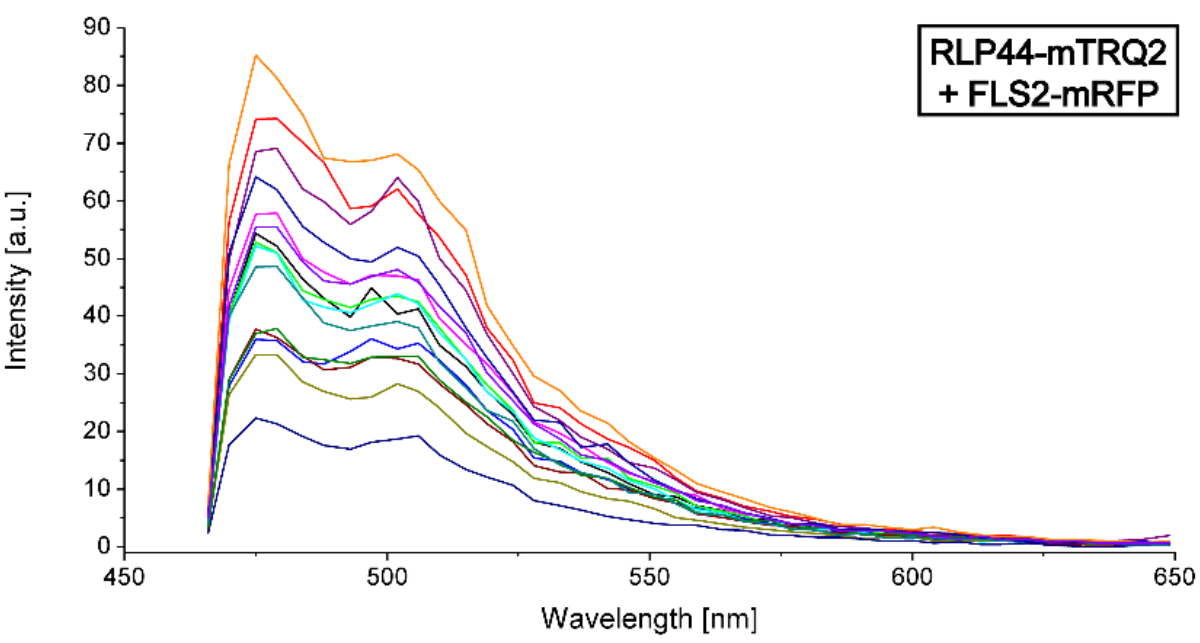

SI Figure 2 | Wavelength-dependent intensity measurements reveal different fusion protein expression levels for different plasma membrane regions in transiently transformed $\mathrm{N}$. benthamiana epidermal leaf cells.

The wavelength-dependent raw intensity of 13 different regions of interest, each, in the plasma membrane of $N$. benthamiana leaf cells after excitation with light of $458 \mathrm{~nm}$ is depicted for RLP44mTRQ2 alone (a), the RLP44-mTRQ2 + FLS2-mRFP (b) and RLP44-mTRQ2 + FLS2-mRFP pair (c). 


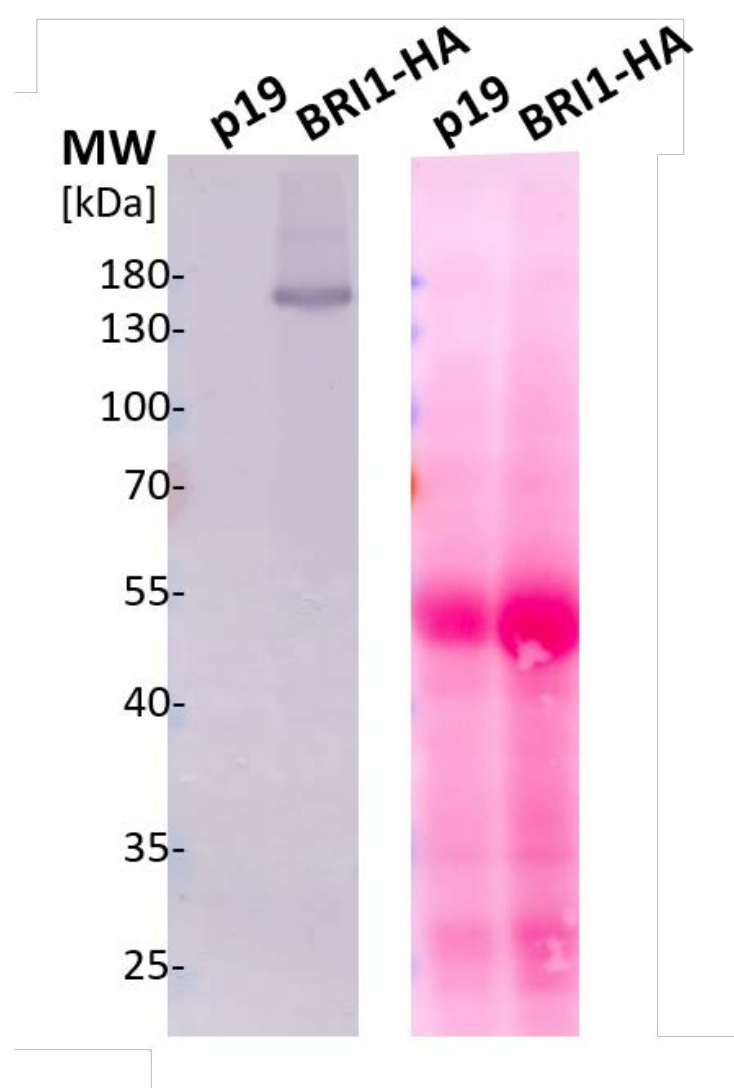

SI Figure 3 | BRI1HA is expressed in transiently transformed N. benthamiana leaf cells.

Western blot (left) and the total protein on the transfer membrane, stained with Ponceau S (right) of $N$. benthamiana leaf extracts prepared two days after transformation with either the silencing inhibitor plasmid p19 (control) or with $\mathrm{p} 19$ and a plasmid coding for BRI1 ${ }^{\mathrm{HA}}$. BRI1 ${ }^{\mathrm{HA}}$ was detected with an HA antibody. The protein size markers are shown to the left. 


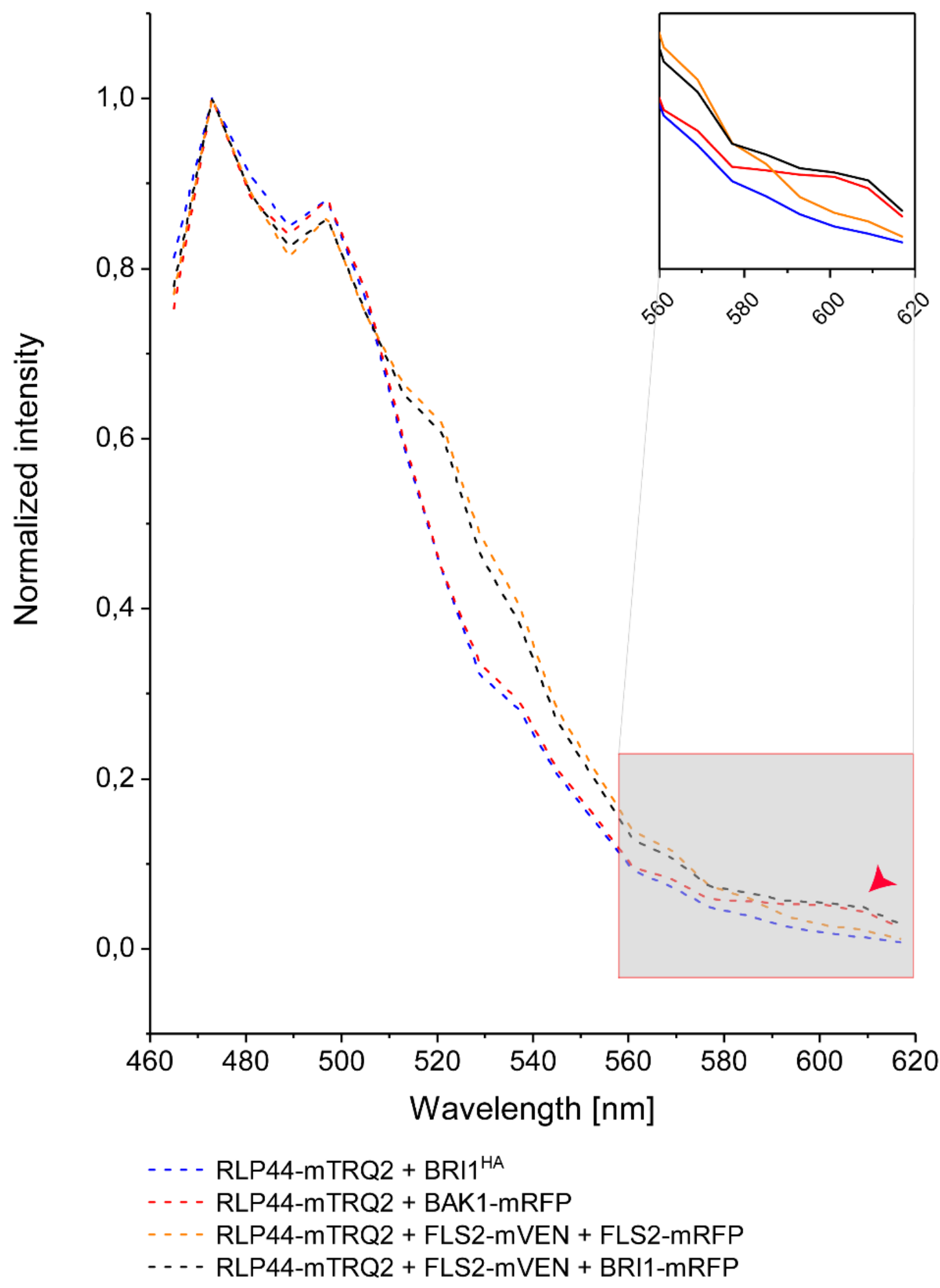

SI Figure 4 | FLS2 does not interfere with the RLP44-related interactions (extension to Fig. 4). Wavelength-dependent normalized fluorescence emission after irradiation of the $N$. benthamiana leaf cells with $485 \mathrm{~nm}$ light for the co-expression of RLP44-mTRQ2 with BRI1 ${ }^{\text {HA }}$ (blue) or with BAK1-mRFP (red) or FLS2-mVEN and FLS2-mRFP (orange) or FLS2-mVEN and BRI1-mRFP4 (black). The FRETrelevant wavelength area is highlighted in the enlarged section. The occurrence of FRET from mTRQ2 to $\mathrm{mRFP}$ is indicated by a red arrow head. 
a

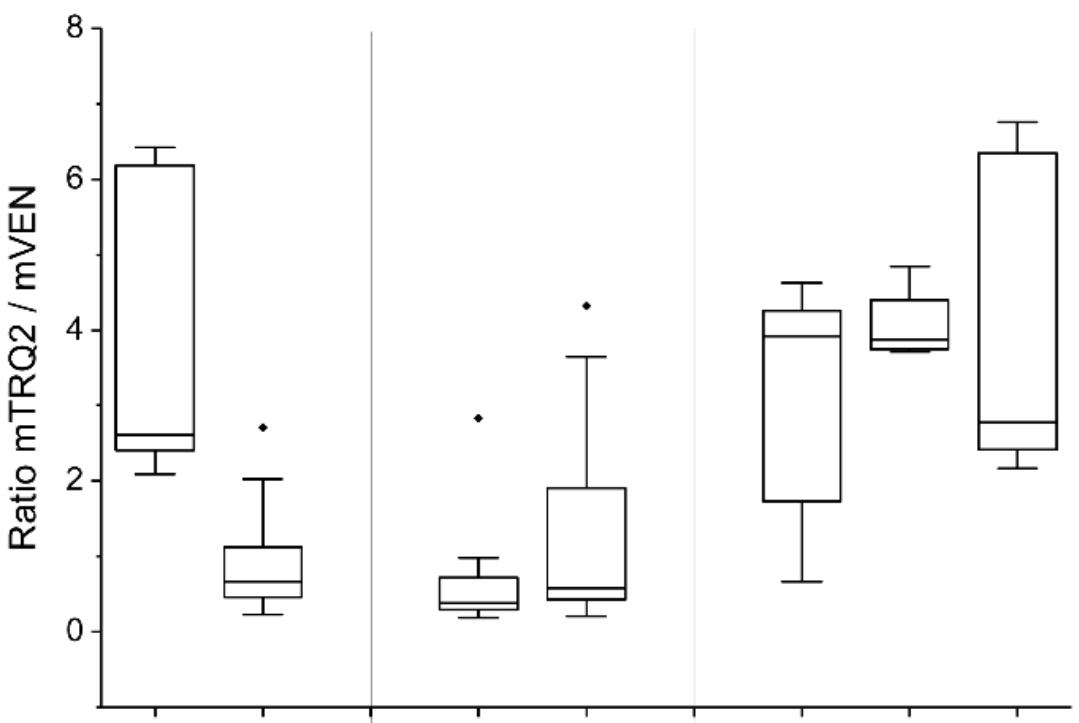

b

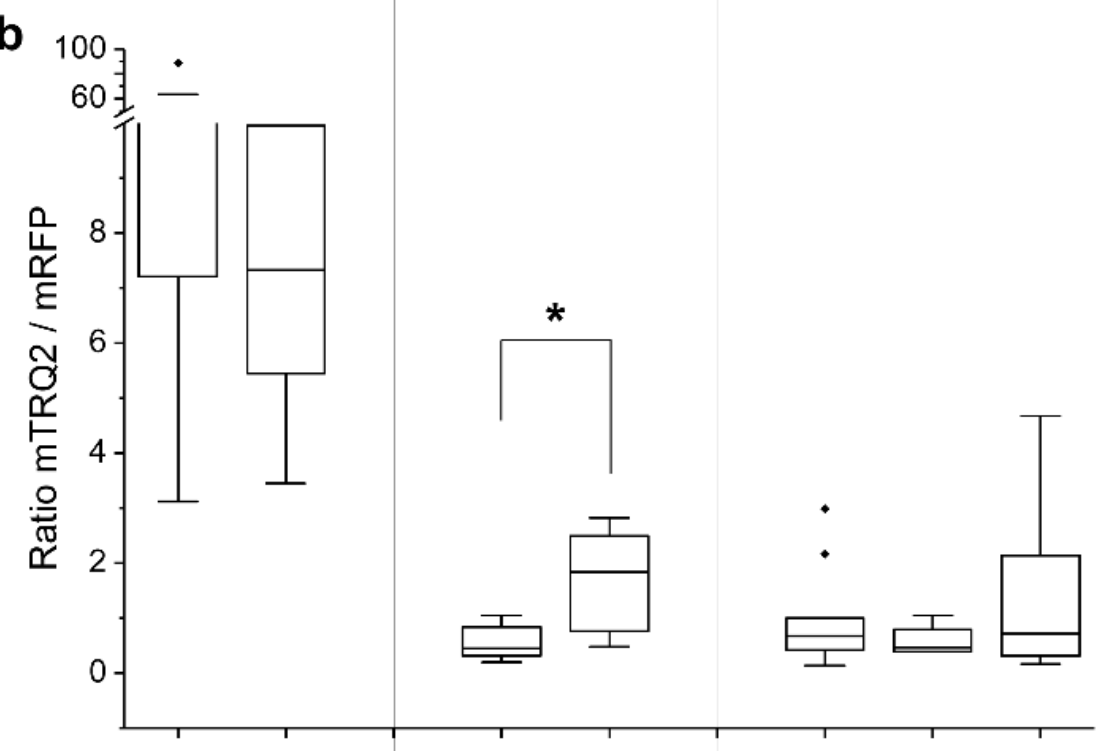

C

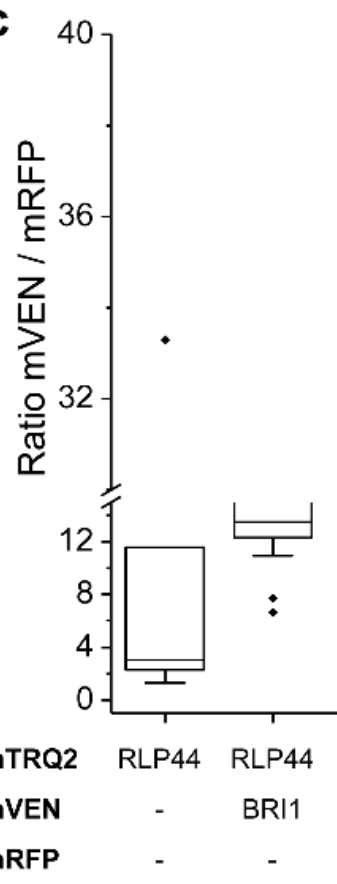

mRFP 
SI Figure 5 | The overall donor to acceptor ratios is not significantly different in the protein fusion arrangements of the FRET-FLIM analysis.

Fluorescence emission ratios for the $\mathrm{mTRQ} 2 / \mathrm{mVEN}$ fusion protein pairs (a), the $\mathrm{mTRQ} 2 / \mathrm{mRFP}$ fusion protein pairs (b) and mVEN/mRFP fusion protein pairs (c) are shown. Data points were left-right scattered (black dots) and combined with boxplot information, permitting outliers.

The ratios for $\mathrm{mTRQ} 2 / \mathrm{mVEN}, \mathrm{mTRQ} 2 / \mathrm{mRFP}$ and $\mathrm{mVEN} / \mathrm{mRFP}$ were tested for significant differences with a two-tailed, all-pair Kruskal-Wallis test followed by a Steel-Dwass post hoc correction. The asterisks marks a significant difference $(p<0.05)$ in the donor-to-acceptor ratios, which was the mTRQ2/mRFP ratio in the RLP44-mRFP4/BRI1-mVEN/BAK1-mRFP and the RLP44-mTRQ2/BRI1$\mathrm{mVEN} / \mathrm{FLS2}$-mRFP arrangements. The boxplots represent all data with the median as a solid line within the box that is restricted by the first quartile ( $25 \%$; lower end) and the third quartile (75\%; upper end). The whiskers show the minimum and maximum value of the data, respectively, that are not defined as outliers (1.5 times the interquartile range). Outliers are indicated as black diamonds. 


\section{References}

Balleza, E., Kim, J. M., \& Cluzel, P. (2018). Systematic characterization of maturation time of fluorescent proteins in living cells. Nature Methods, 15(1), 47-51. https://doi.org/10.1038/nmeth.4509.

Bunt, G. \& Wouters, F. S. (2017). FRET from single to multiplexed signaling events. Biophysical reviews 9, 119-129; 10.1007/s12551-017-0252-z.

Campbell, R. E., Tour, O., Palmer, A. E., Steinbach, P. A., Baird, G. S., Zacharias, D. A., \& Tsien, R. Y. (2002). A monomeric red fluorescent protein. Proceedings of the National Academy of Sciences of the United States of America, 99(12), 7877-7882. https://doi.org/10.1073/pnas.082243699

Cranfill, P. J., Sell, B. R., Baird, M. A., Allen, J. R., Lavagnino, Z., Gruiter, H. M. de, . . Piston, D. W. (2016). Quantitative assessment of fluorescent proteins. Nature Methods, 13(7), 557-562. https://doi.org/10.1038/nmeth.3891

Goedhart, J., Stetten, D. v., Noirclerc-Savoye, M., Lelimousin, M., Joosen, L., Hink, M. A., . . Royant, A. (2012). Structure-guided evolution of cyan fluorescent proteins towards a quantum yield of 93\%. Nature Communications, 3, 751. https://doi.org/10.1038/ncomms1738

Koushik, S. V., Blank, P. S. \& Vogel, S. S. (2009). Anomalous surplus energy transfer observed with multiple FRET acceptors. PloS One 4, e8031; 10.1371/journal.pone.0008031.

Kremers, G.-J., Goedhart, J., van Munster, E. B., \& Gadella, T. W. J., JR. (2006). Cyan and yellow super fluorescent proteins with improved brightness, protein folding, and FRET Forster radius. Biochemistry, 45(21), 6570-6580. https://doi.org/10.1021/bi0516273.

Liu, J., Lu, Y. (2002). FRET study of a trifluorophore-labeled DNAzyme. J. Am. Chem. Soc., 124(51), 15208-15216. https://pubs.acs.org/doi/10.1021/ja027647z.

Nagai, T., Ibata, K., Park, E. S., Kubota, M., Mikoshiba, K., \& Miyawaki, A. (2002). A variant of yellow fluorescent protein with fast and efficient maturation for cell-biological applications. Nature Biotechnology, 20(1), 87-90. https://doi.org/10.1038/nbt0102-87

Scott, B. L., \& Hoppe, A. D. (2015). Optimizing fluorescent protein trios for 3-Way FRET imaging of protein interactions in living cells. Scientific Reports, 5, 10270 EP -.

https://doi.org/10.1038/srep10270 